\title{
Asymmetric Doherty Power Amplifier Designed Using Model-Based Nonlinear Embedding
}

\author{
Haedong Jang, Member, IEEE, Patrick Roblin, Member, IEEE, Christophe Quindroit, Yiqiao Lin, and \\ Robert D. Pond
}

\begin{abstract}
A novel procedure is introduced for designing Doherty amplifiers using the model-based nonlinear-embedding technique. First, the Doherty intrinsic load-matching network is designed at the transistor current-source reference plane with the main and auxiliary devices interconnected. Identical devices with different biasing are used for realizing an asymmetric Doherty implementation with 9-dB back-off. The required multiharmonic impedances at the package planes are then obtained using the embedding device model for both devices, and the complex load impedance at the fundamental is projected back to resistive loads using an offset line. An even-number multisection impedance transformer and a reduced drain voltage of the main amplifier are used to design the asymmetric Doherty load network while providing the necessary loads to the main and auxiliary devices. The optimization of the drain efficiency and gain curves of the asymmetric Doherty operation for the proposed design is further investigated by adjusting the auxiliary gate-bias. An efficiency above $50 \%$ over an 11-dB power range is experimentally observed with 41.8-dBm peak output power using continuous wave $(\mathrm{CW})$ at $2 \mathrm{GHz}$. Using a dual-input implementation of the designed Doherty power amplifier (PA), a systematic dual-input $\mathrm{CW}$ characterization of the Doherty operation is performed to establish the relative auxiliary-to-main phase offsets and power offsets yielding a maximum efficiency under constant gain. From this dual-input characterization, it is found that the optimal gate bias for single-input Doherty operation is the one for which the constant-gain maximum efficiency is achieved for a quasi-constant auxiliary-to-main input power ratio corresponding to the one implemented in the input divider in the single-input Doherty PA.
\end{abstract}

Index Terms-Asymmetric, Doherty, embedding, load modulation, nonlinear, power amplifier (PA).

\section{INTRODUCTION}

$\mathbf{H}$ IGH-DATA-RATE communication environments require spectrally efficient modulation schemes with a high peak-to-average power ratio (PAPR). Typical RF power amplifiers (PAs) have peak efficiency only at peak power, and the efficiency is reduced at backed-off power levels, resulting

Manuscript received July 02, 2014; revised September 14, 2014 and October 16, 2014; accepted October 24, 2014. Date of publication November 20, 2014; date of current version December 02, 2014. This work was supported in part by the National Science Foundation under Grant ECS 1129013. This paper is an expanded version from the IEEE MTT-S International Microwave Symposium, Tampa Bay, FL, USA, June 1-6, 2014.

H. Jang, P. Roblin, Y. Lin, and R. D. Pond are with the Department of Electrical and Computer Engineering, The Ohio State University, Columbus, $\mathrm{OH}$ 43210 USA (e-mail: jang.131@osu.edu; roblin@ece.osu.edu).

C. Quindroit is with LPA Concepts, 33650 Martillac, France.

Color versions of one or more of the figures in this paper are available online at http://ieeexplore.ieee.org.

Digital Object Identifier 10.1109/TMTT.2014.2366130

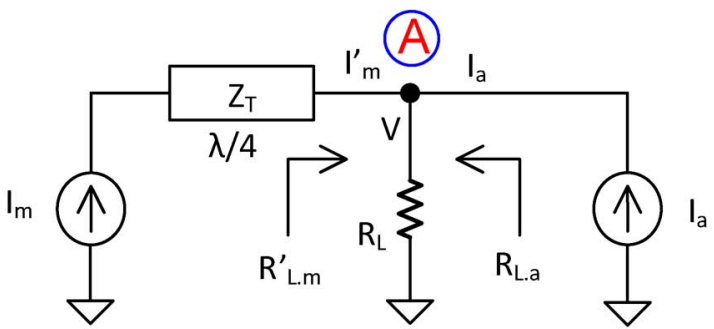

(a)

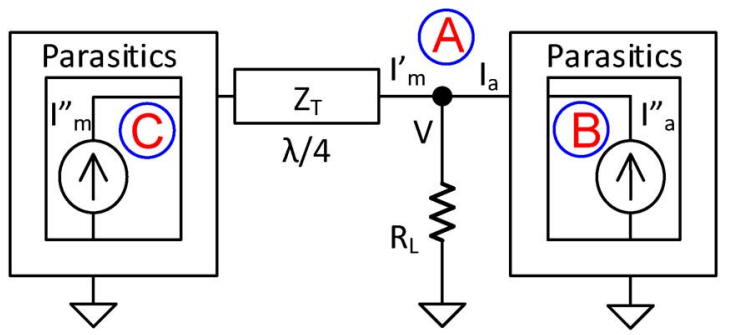

(b)

Fig. 1. Conceptual Doherty amplifier (a) without parasitics and (b) with parasitics. The effective loads seen by the main and auxiliary amplifiers are varied by the current contributions from each amplifier.

in a degraded average efficiency. Doherty amplifiers have attracted RF power amplifier designers due to their improved average efficiency realized with a relatively simple structure [1], [2]. The conventional Doherty amplifier provides only a 6-dB back-off level while the signals such as long-term evolution (LTE) or multiple-carrier wideband code-division multiple-access (WCDMA) exhibit PAPR over $10 \mathrm{~dB}$. Asymmetric Doherty amplifiers have been demonstrated to extend the back-off range while yielding higher average efficiency [2]-[5].

A conceptual diagram of the Doherty amplifier is shown in Fig. 1(a). The main and auxiliary amplifiers are modeled as current sources. The power-combining junction, marked as A in the figure, is ideally located at the current-source reference plane of the auxiliary amplifier [6]. The modulated load $R_{L . m}^{\prime}$ controlled by the auxiliary current $I_{a}$, is inverted by a quarter-wave transformer of impedance $Z_{T}$ and applied to the main-device current source (intrinsic $I-V$ characteristics) at the current-source reference plane.

Accurately providing modulated loads to the devices is critical for obtaining Doherty operation. However, high-power packaged devices have non-negligible linear/nonlinear parasitic components, as shown in Fig. 1(b). The junction A is no longer the same as node $\mathrm{B}$, and the electrical distance between 
junctions $\mathrm{A}$ and $\mathrm{C}$ is longer than a quarter wavelength. Proper matching network and impedance inverters are thus required to absorb those parasitic components. Introducing additional transmission lines between junction A and node B was proposed in [6] to bring the auxiliary device intrinsic node out of the device while accounting for the dominating parasitic components. Typically, a significant amount of simulation optimization or device characterization efforts when device models are not available is required from designers to compensate for the parasitic components.

A more systematic method would be to start the design at the current-source reference plane and then predict the external loads or excitations at the fundamental or harmonics required to maintain the intended intrinsic operation using the hybrid nonlinear/linear embedding/de-embedding techniques [8]-[14]. An embedding device model has been recently reported for the Angelov model which facilitate this approach when an Angelov device model is available [7]. Applying this technique for designing the matching network of amplifiers using a single device can be straightforward. However, the design of a Doherty amplifier would be expected to be more complicated than that of a single-device amplifier because of the interaction between the main and auxiliary transistors via their parasitic networks, matching network, and the Doherty combiner network. Furthermore, the nonlinear embedding technique can only be applied after the intrinsic operation of both transistors has been first defined.

To address the above issues and greatly simplify the design procedure, we propose to first implement a Doherty PA using only the current sources of both devices. The advantage of synthesizing first the Doherty operation at the current planes as proposed in this paper compared with the conventional design approach with the full device model is that the interaction between the two transistors can be optimized to implement the Doherty load-modulation matching the device $I-V$ characteristics. The matching network and the offset lines then required to implement this effective Doherty operation at the current source plane can then be synthesized using the recently proposed nonlinear-embedding device model. To this order, the authors developed a modified Doherty design procedure using two ideal transformers for the intrinsic Doherty load-matching network design before the nonlinear embedding technique is applied. This approach was demonstrated experimentally in the first-pass design and realization of a $16.7-\mathrm{W}$ peak-power asymmetric GaN Doherty PA [5]. A 51.86\% average drain efficiency was observed after linearization maintaining $-51.46-\mathrm{dBc}$ adjacent channel power ratio (ACPR) excited for $10-\mathrm{MHz}$ bandwidth LTE signals with 8.85-dB PAPR. In this expanded version of the paper, the step-by-step design procedure is presented in detail, and the Doherty design equations are further generalized for arbitrary maximum current ratios and maximum voltage ratios for the main and auxiliary transistors. Further new work reported in this paper is also described next.

The turn-on time of the auxiliary amplifier can be controlled by the gate bias. CW large-signal measurements were conducted at multiple auxiliary amplifier gate bias points to investigate the Doherty operation realized through the proposed design procedure. Of particular interest is the observation of both the efficiency at various power back-off and the gain flatness. However, a gate bias variation in class-C operation affects the peak current of the auxiliary amplifier requiring a corresponding power-division ratio adjustment of the input power divider [15]. The Doherty amplifier in [5] has a fixed power-ratio divider at the input. Thus, to address this issue in this expanded version of [5], a dual-input Doherty amplifier without the fixed power-ratio divider [16], [17] was also designed and fabricated using the same nonlinear embedding technique. The operations with varied auxiliary gate bias conditions are then investigated without the fixed power-division constraint. A systematic characterization method for the dual-input Doherty amplifier is presented in the high-power region where load-modulation is taking place. An algorithm for extracting the optimal constant-gain maximum efficiency (CGME) from the systematic characterization data is also proposed. The optimal operation with varied auxiliary gate-bias conditions is then investigated and compared with the single-input fixed-power-ratio Doherty PA operation reported in [5].

Even-section quarter-wave transformers were introduced in [5] to facilitate the use of identical devices for both the main and auxiliary amplifiers. It provides the necessary loads to the devices so as to realize the adjustable load-modulation matching. In this expanded work, the bandwidth characteristic provided by the transformers is analyzed. It is established that the major bandwidth constraint is dominated by the Doherty quarter-wave impedance inverter instead of the added even-section impedance transformers. Further bandwidth improvement can thus be pursued by adopting advanced wideband inverter structures such as the ones proposed in [18]-[20].

The adjustable matching network for asymmetrical Doherty amplifier design using identical devices is briefly reviewed in Section II. The Doherty amplifier design process using nonlinear embedding is elaborated in Section III together with the bandwidth analysis of the even-section transformers. The single-input Doherty amplifier measurements results are analyzed for the auxiliary gate bias adjustment in Section IV. The dual-input Doherty amplifier is introduced in Section V together with the systematic characterization method, the CGME algorithm, and an analysis of the measured results. Finally, a summary of the results obtained is presented in Section VI.

\section{MATChING NeTWORK FOR ASYMMETRIC DOHERTY PA USING IDENTICAL DEVICES}

The efficiency of Doherty PAs is well derived in [21] assuming that the auxiliary amplifier is linear and approaching a class-B operation condition. The efficiency for the high-power region where the load-modulation is taking place can be approximated by

$$
\eta_{\text {high }}=\frac{\pi(n+1)\left|v_{\text {in.norm }}\right|^{2}}{4\left[(n+2)\left|v_{\text {in.norm }}\right|-1\right]}, \quad \frac{1}{n+1} \leq\left|v_{\text {in.norm }}\right| \leq 1
$$

which can be re-derived using the power-ratio notation $n$ between the auxiliary and main amplifiers, i.e., the auxiliary amplifier provides $n$ times more power than the main amplifier. In (1), $v_{\text {in.norm }}$ is the RF input voltage amplitude normalized to the peak input amplitude of the example input signals de-crested 


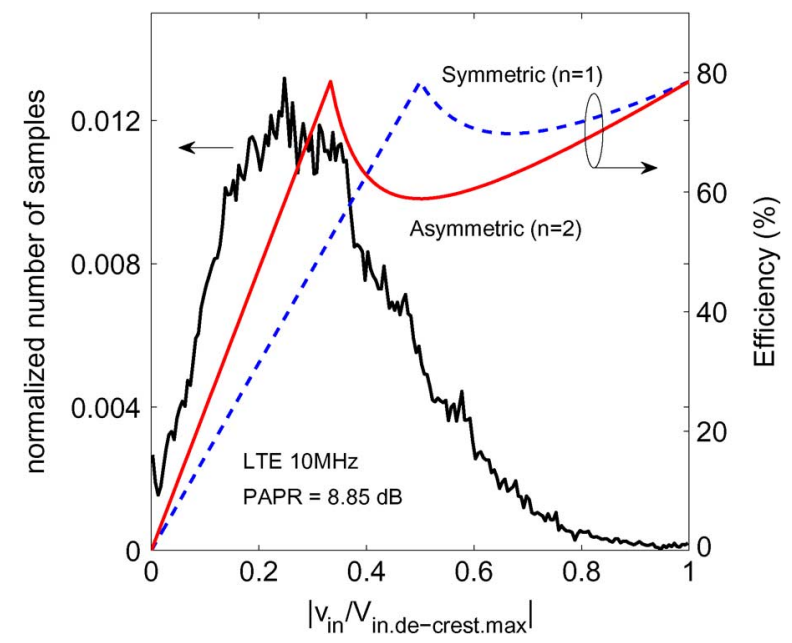

Fig. 2. Asymmetric Doherty amplifier efficiency.

to have $8.85-\mathrm{dB}$ PAPR. $\alpha$ in [21] is equivalent to $1 /(n+1)$. The efficiency in the lower power region, where only the main amplifier is turned on, is assumed to be the same as a class-B power amplifier as shown in Fig. 2 [21], [24]. The efficiency curve can be shifted to the left by using $n$ larger than 1, resulting in higher average efficiency for high peak-to-average signals. For example, the efficiency calculated using a LTE $10-\mathrm{MHz}$ signal with 8.85-dB PAPR for the asymmetric structure $(n=2)$ shows $7.7 \%$ higher average efficiency than the symmetric case $(n=1)$.

The effective load seen by each amplifier in Fig. 1(a) is varied by the current contribution of the other amplifier. Those loads can be expressed using the power ratio $n$ assuming both currents are in-phase. The power delivered from the auxiliary amplifier side is assumed to be $n$ times larger than the power delivered from the main amplifier side $\left(P_{a}=n P_{m}^{\prime}\right)$. Since the voltage $V$ at node $\mathrm{A}$ is common, the auxiliary current is $n$ times larger than the main $\left(I_{a}=n I_{m}^{\prime}\right)$. The voltage developed at node A by the summed currents is $V=\left(I_{m}^{\prime}+I_{a}\right) R_{L}$. Therefore, the effective loads seen from each amplifier are

$$
\begin{aligned}
R_{L . m}^{\prime} & =\frac{V}{I_{m}^{\prime}}=(n+1) R_{L} \\
R_{L . a} & =\frac{V}{I_{a}}=\frac{(n+1)}{n} R_{L} .
\end{aligned}
$$

\section{A. Preferred Loads by Devices}

An optimal load $R_{\text {opt }}$ for the peak power is decided by the device maximum current $I_{\max }$ and the maximum voltage $V_{\text {max }}$ considering the breakdown voltage as illustrated in Fig. 3. The solid lines are ideal class-B load lines at three different power levels. The efficiency during load modulation can be shown to remain constant by selecting loads inversely proportional to power levels [24] as follows:

$$
\eta=\frac{\pi\left(V_{d d}-V_{\mathrm{on}}\right)}{4 V_{d d}}
$$

where $V_{\text {on }}$ is the knee voltage in the figure. However, for practical devices, it is observed that the efficiency at low powers with high impedances degrades in the presence of on-resistance

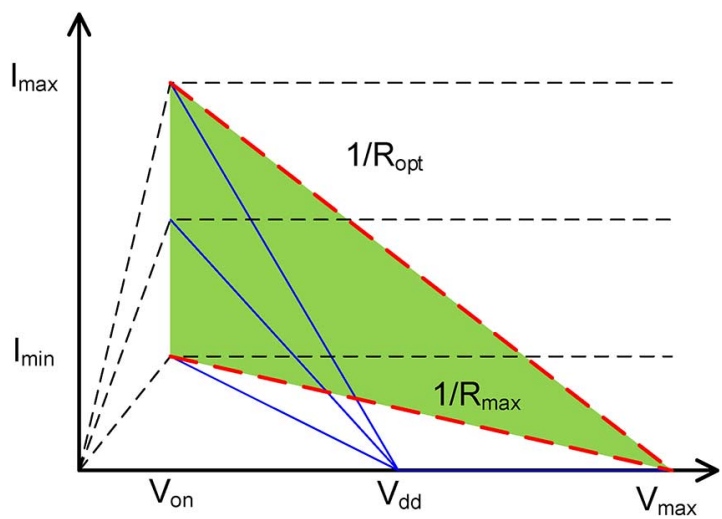

Fig. 3. Preferred loads for the auxiliary device under ideal class-B operation are shown in the shaded region.

[7]. Therefore, the load-modulation range is limited by the device characteristics to a maximum practical load $R_{\max }$. Thus, the preferred load modulation range for both of the devices is

$$
\begin{aligned}
R_{\text {opt } . \mathrm{m}} & \leq R_{L . m . \text { preferred }} \leq R_{\max . m} \\
R_{\text {opt }} & \leq R_{L . a . \text { preferred }} \leq \infty .
\end{aligned}
$$

The load seen by the main amplifier through the quarter-wave impedance inverter of the Doherty amplifiers are $(n+1)^{2} R_{L}$ and $(n+1) R_{L}$ for the backed-off power level and the peak power level, respectively. Thus, designers can choose those loads to fall into the devices' preferred range.

In the symmetrical Doherty design, the loads seen by the main and auxiliary amplifiers at the peak power are the same $R_{L . m}^{\prime}=R_{L . a}$ when $n=1$ from (2). However, in the asymmetric Doherty design where $n \neq 1$, the two loads seen by the both amplifiers are different $\left(R_{L . m}^{\prime} \neq R_{L . a}\right)$ which is not desirable when using identical devices. Therefore, in this work, additional impedance transform ratios $(\alpha, \beta)$ are introduced for both the main and auxiliary amplifiers to allow for using identical devices. Among the many possible transformer implementations, multisection impedance transformers were introduced in this work as shown in Fig. 4. Multisection impedance transformers provide a convenient way of implementing the impedance transformation by choosing the characteristic impedances of the lines with broader bandwidth and also controlled passband shaping by using binomial polynomials or Chebyshev polynomials. It should be noted that the quarter-wave transformers introduce an additional phase shift of $e^{-j k \pi}$, where the $k$ is the number of sections used. Therefore, an even number of sections should be used to maintain the proper Doherty load modulation.

\section{B. Peak Power Rescaling}

The auxiliary amplifier provides $n$ times more power than the main amplifier at the peak power. Therefore typically a larger device is used for the auxiliary of the asymmetric Doherty power amplifiers [3]. However, as shown in Appendix I, different voltage ratios $\gamma_{V}$ and current ratios $\gamma_{I}$ can be found to yield the same power ratio $n$ if we select them to verify $n=1 /\left(\gamma_{I} \gamma_{V}\right)$. In this work, a reduced drain voltage $\left(\gamma_{V}=0.5\right)$ was used to reduce the main amplifier power as shown in Fig. 5 while using identical devices $\left(\gamma_{I}=1\right)$. 


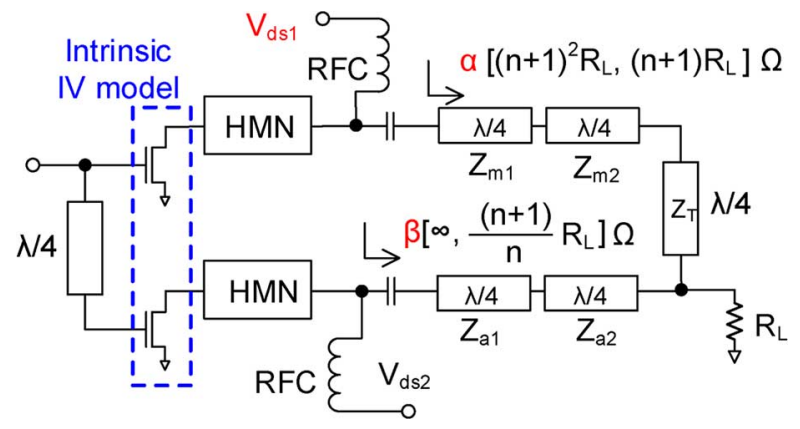

Fig. 4. Adjustable Doherty amplifier-matching network at the current-source reference plane. HMN stands for harmonic matching network. Harmonics are ideally terminated at the current-source reference plane.

When using a reduced drain voltage for the main amplifier, it is still possible to have the main and auxiliary loads varying in the preferred load range of the device with the help of two transformers. The impedance variation ratio can be defined as

$$
\gamma_{V}=\frac{V_{d d . \text { reduced }}-V_{o n}}{V_{d d}-V_{\text {on }}}
$$

leading to the following effective optimal and maximum loads for the main amplifier when identical devices $\left(\gamma_{I}=1\right)$ are used:

$$
R_{\mathrm{opt} . m}=\gamma_{V} R_{\mathrm{opt}} \quad R_{\max . m}=\gamma_{V} R_{\max } .
$$

As indicated in Fig. 4, the loads seen by the main and auxiliary amplifiers vary under load modulation in the range

$$
\begin{aligned}
\alpha(n+1) R_{L} & <R_{L . m}<\alpha(n+1)^{2} R_{L} \\
\beta \frac{n+1}{n} R_{L} & <R_{L . a}<\infty .
\end{aligned}
$$

The design equations can then be expressed in terms of the optimal and maximum loads as follows:

$$
\begin{aligned}
R_{\mathrm{opt} . m} & =\alpha(n+1) R_{L} \\
R_{\max . m} & \geq \alpha(n+1)^{2} R_{L} \\
R_{\mathrm{opt}} & =\beta \frac{n+1}{n} R_{L} .
\end{aligned}
$$

From these equations, we deduced that the max load must verify $R_{\max . m} \geq(n+1) R_{\text {opt. } m}$. Equations (6)-(10) still hold for nonunity $\gamma_{I}$, as can be verified using the general equation $R_{\text {opt. } m}=$ $\gamma_{V} / \gamma_{I} R_{\text {opt }}$. The transformer ratio $\beta$ must verify $\sqrt{\beta}=\sqrt{\alpha} / \gamma_{V}$ while we have $\gamma_{I}=1 /\left(n \gamma_{V}\right)$ (see Appendix I). In summary, designers can choose the design parameters $R_{L}, \alpha$, and $\beta$ to provide the preferred load ranges to the devices for a given power ratio $n$ [5] while adjusting the maximum voltage and current ratios $\gamma_{V}$ and $\gamma_{I}$ according to (see Appendix I)

$$
\gamma_{V}=\frac{\left|V_{m \cdot \max }\right|}{\left|V_{a \cdot \max }\right|}=\sqrt{\frac{\alpha}{\beta}} \quad \gamma_{I}=\frac{\left|I_{m \cdot \max }\right|}{\left|I_{a \cdot \max }\right|}=\frac{1}{n} \sqrt{\frac{\beta}{\alpha}}
$$

The generalized asymmetric Doherty design equations reduce to the classic asymmetric Doherty design equations [3] when selecting $\gamma_{V}=\alpha=\beta=1$.

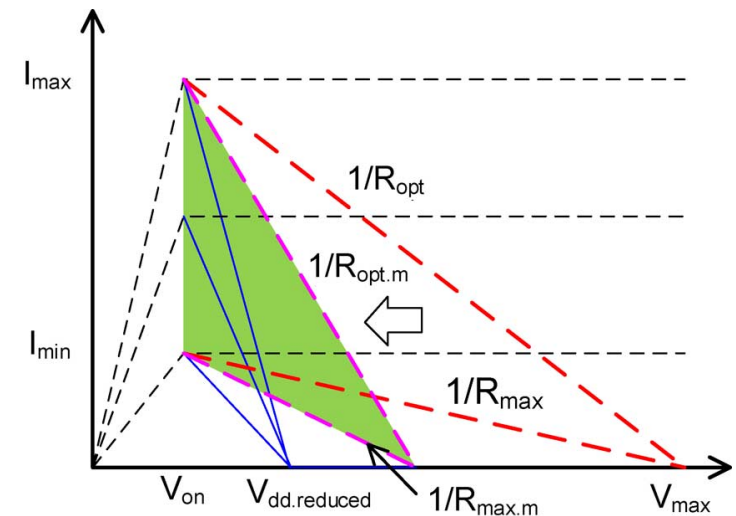

Fig. 5. Preferred loads (shaded area) for the main device are effectively reduced by the lowered drain bias voltage.

\section{INTRINSIC DOHERTY LOAD-NETWORK DESIGN}

\section{A. Intrinsic Doherty Load-Network Design}

The model-based nonlinear embedding technique from [7] allows designers to start the PA design at the current-source reference plane using the intrinsic $I-V$ model while accounting for the leading memory effects such as group delay and selfheating. With this approach, designers can tailor the intrinsic operation modes as close as possible to the ideal waveform using ideal intrinsic harmonic terminations. Once the intrinsic operation is defined using a certain number of harmonics, the external multiharmonic voltages and currents at the external (package) reference planes sustaining it are calculated in a single simulation using the embedding device model accounting for all of the device nonlinear/linear parasitic/charge components [7]. Using those external voltages and currents, the external harmonic terminations and input excitations to keep the intrinsic intended operation can be predicted [8] as follows:

$$
\begin{aligned}
Z_{L}(n \omega) & =-\frac{V_{\mathrm{DS}}^{e}(n \omega)}{I_{D}^{e}(n \omega)} \\
Z_{S}(n \omega) & =-\frac{V_{\mathrm{GS}}^{e}(n \omega)}{I_{G}^{e}(n \omega)}, \quad n>2 \\
Z_{S}(\omega) & =\operatorname{conj}\left(\frac{V_{\mathrm{GS}}^{e}(\omega)}{I_{G}^{e}(\omega)}\right)
\end{aligned}
$$

where $n$ is the harmonic index, $V_{\mathrm{DS}}^{e}(n \omega), I_{D}^{e}(n \omega), V_{\mathrm{GS}}^{e}(n \omega)$, and $I_{G}^{e}(n \omega)$ are the predicted external drain and gate voltages and currents at corresponding harmonic components. Currents are assumed to flow into the device. The fundamental input can be conjugate matched using the predicted input impedance in large-signal condition [8], [25]. For the harmonic, a small load renormalization is required as usually the load reflection coefficients $\Gamma_{L}(n \omega)$ are slightly outside the Smith Chart [7]. Using this technique, the design is significantly simplified removing the need for time-consuming harmonic source/load pull measurements or simulations.

The intrinsic $I-V$ model of a $15-\mathrm{W}$ peak power GaN device (CGH27015F, CREE Inc.) was used for both main and auxiliary amplifiers. The drain bias of the main device was reduced from $28 \mathrm{~V}$ to $14 \mathrm{~V}$ and the gate voltage was $-2.9 \mathrm{~V}$, resulting 
in 89-mA intrinsic quiescent current. The second harmonic was shorted and the third kept open for class-F operation. The auxiliary device was bias at $28 \mathrm{~V}$ for the drain and $-3.9 \mathrm{~V}$ for the gate, and the second and third harmonics were shorted for class-C operation. The load network in Fig. 4 was designed using $R_{L}=12.27 \Omega, \alpha=0.5$, and $\beta=1.5$. The methodology followed is explained next.

The device characterization for the CGH27015F in [7] showed that output loads of $R_{\mathrm{opt}}=27.6 \Omega$ to $R_{\max }=85.8 \Omega$ were optimal for $9.6 \mathrm{~W}$ and $3.0 \mathrm{~W}$ output power, respectively. About $57 \%$ power-added efficiency (PAE) was reported at the peak power and was maintained over high-power range at class-B operating condition, and it was gradually degraded down to $51 \%$ at low powers. In this design, it is targeted to have $10 \mathrm{~W}$ provided by the auxiliary amplifier and $5 \mathrm{~W}$ by the main amplifier at the peak power in order to obtain the desired 9.54-dB back-off $(n=2)$. Note that the power ratio $n$ is a design parameter which can be decided considering the PAPR of the signals of interests. In this work, $n=2$, which is 9.54-dB back-off, was chosen for demonstration purposes, considering high PAPR signals such as WCDMA and LTE signals.

Note that the load variation is approximately three times which is necessary for 9.54-dB back-off asymmetric design $(n+1=3)$. The three unknown variables $\alpha, \beta$, and $R_{L}$ were decided using the load range from the device characteristics and (8)-(10). First of all, the auxiliary amplifier peak power was optimized using (10). Substituting (10) into (6), we obtain

$$
\gamma_{V}^{2} n R_{\mathrm{opt}}<R_{L . m}<\gamma_{V}^{2} n(n+1) R_{\mathrm{opt}}
$$

This selected range of load for the main PA should be included in the preferred ranged defined by (5) as

$$
\gamma_{V}^{2}\left[n R_{\mathrm{opt}}, n(n+1) R_{\mathrm{opt}}\right] \subset \gamma_{V}\left[R_{\mathrm{opt}}, R_{\mathrm{max}}\right]
$$

yielding the requirement

$$
\gamma_{V}\left[n R_{\mathrm{opt}}, n(n+1) R_{\mathrm{opt}}\right] \subset\left[R_{\mathrm{opt}}, R_{\mathrm{max}}\right] .
$$

For our particular design, this gives

$$
\sqrt{\frac{\alpha}{\beta}}[55.2 \Omega, 165.6 \Omega] \subset[27.6 \Omega, 85.8 \Omega] .
$$

using $\gamma_{V}=\sqrt{\alpha / \beta}$. Among many solutions, $\alpha=$ $0.4, \beta=1.5$ can be chosen resulting in a load range $\gamma_{V}^{2}\left[n R_{\text {opt }}, n(n+1) R_{\text {opt }}\right]$ of $\gamma_{V}[28.5 \Omega, 85.5 \Omega]$ within the required range $\gamma_{V}[27.6 \Omega, 85.8 \Omega]$ but in the practical design $\alpha=0.5$ was chosen for ease of the design of the even section transformer. Therefore, more saturated operation is expected with higher impedance loads. Finally the load $R_{L}$ is evaluated from (10) to be $R_{L}=12.27 \Omega$.

The designed intrinsic load lines are shown in Fig. 6(a) and (b) for the main and auxiliary devices, respectively. The main amplifier load lines with swept power exhibit clear switching operation in class-F mode. The auxiliary amplifier load lines show class-C operation in the linear $I-V$

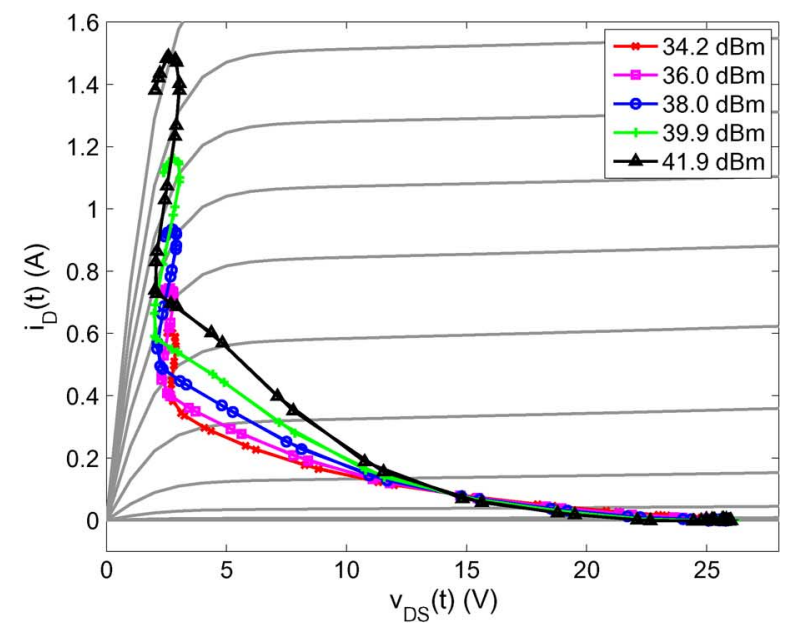

(a)

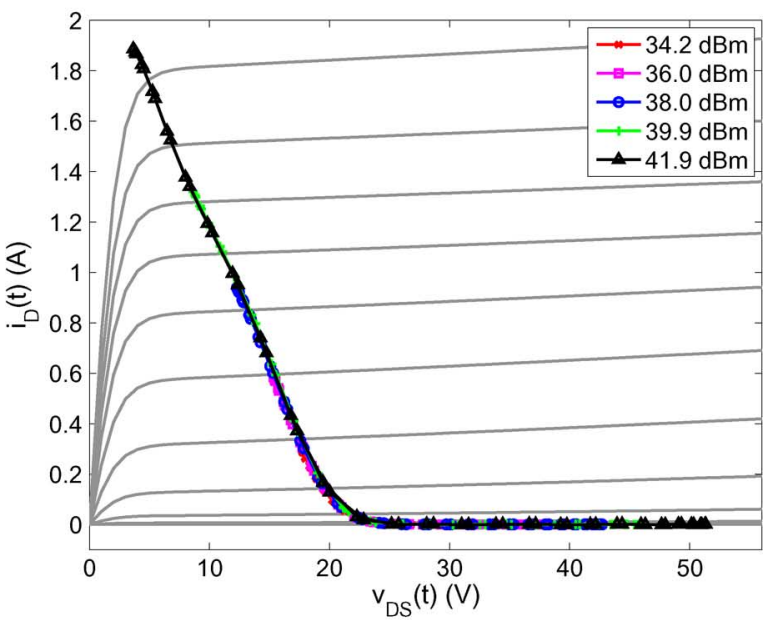

(b)

Fig. 6. Intrinsic load lines of (a) class-F main amplifier and (b) class-C auxiliary amplifier for varied input drive powers.

region. The reduced drain voltage swing of the main amplifier can be noticed when compared with the auxiliary amplifier.

The key point is that the Doherty load network is intrinsically designed using the $I-V$ model without considering the parasitic components at the initial design stage. This allows the definition of an effective intrinsic Doherty operation through the interaction of both the main and auxiliary amplifiers before even considering the parasitic and compensation network design. This intrinsically defined Doherty operation can be then used by the nonlinear embedding technique, as will be detailed in the next section.

\section{B. Intrinsic to Extrinsic Mode Projection Using Nonlinear Embedding}

The intrinsic operations defined in the previous section for both the main and auxiliary amplifiers were individually projected to the package reference planes, as shown in Fig. 7. The intrinsic fundamental and harmonics are shown with gray dots. The intrinsic loads are on the real load line and the harmonics were ideal short $\left(\Gamma_{L . m \text {.int }}(2 \omega)=-1\right)$ and open $\left(\Gamma_{L . m . \text { int }}(3 \omega)=1\right)$ positions. The projected fundamental and harmonic loads through nonlinear/linear parasitics and 


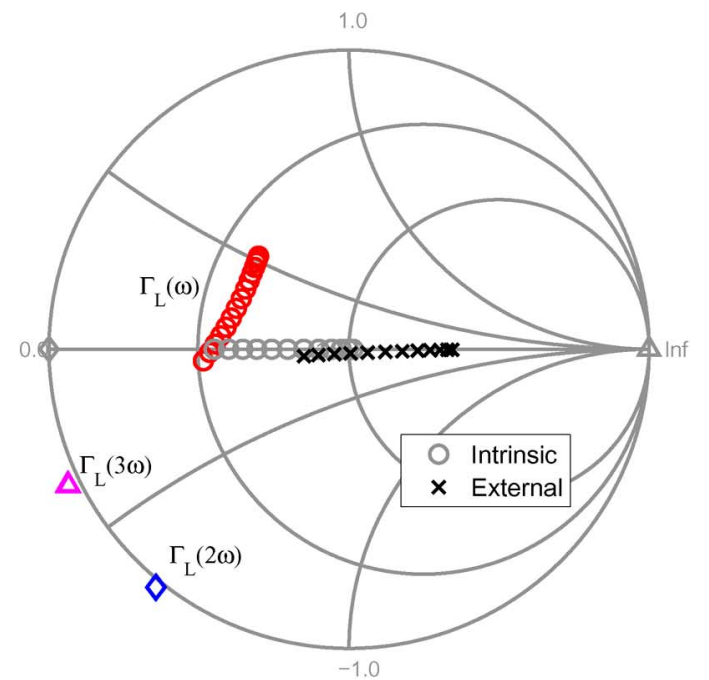

(a)

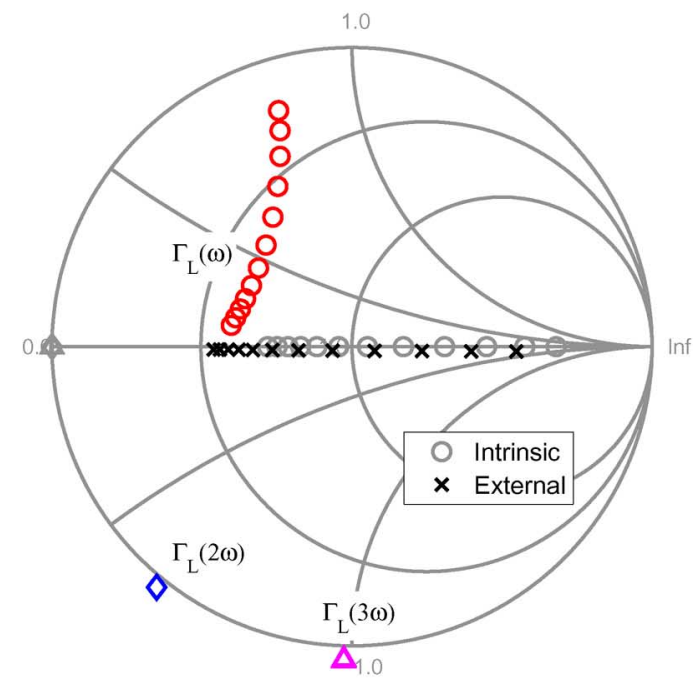

(b)

Fig. 7. $\Gamma_{L}(n \omega)$ obtained at the current source reference planes (gray circles), the package reference planes (red circles), and the Doherty combiner planes after the compensation network (black crosses) for the (a) main and (b) auxiliary amplifiers at $2 \mathrm{GHz}$.

package are also indicated in the figure. It can be noticed that the projected third-harmonic locations for the main and auxiliary are different due to the difference intrinsic class-F and class- $\mathrm{C}$ operation, as expected. It also can be noticed that the projected harmonic loads through the lossy parasitic network are slightly out of the Smith chart [7]. Considering practically realizable passive matching network, the harmonic terminations can be renormalized using the maximum available harmonic terminations [7]

$$
\Gamma_{L}^{\prime}(n \omega)=\frac{\Gamma_{L}(n \omega)}{\left|\Gamma_{L}(n \omega)\right|} \cdot \Gamma_{L . \max . \text { available }}(n \omega)
$$

Therefore, the intrinsic operation modes are no longer ideal due to the lossy harmonic terminations and the performance is expected to be slightly degraded. This slight degradation could be partially compensated using harmonic injection techniques [26]-[28] at the cost of increased circuit complexity [7].

\section{Harmonic Terminations and Offset Line Design}

It can be noted in Fig. 7 that the required external harmonic loads for the asymmetric Doherty amplifier at the package reference plane defined using the nonlinear embedding technique are independent of the load modulation. The harmonics can then be terminated using microstrip open (or short) stubs which have $\lambda / 12$ and $\lambda / 8$ lengths for the third and second harmonics, respectively, as illustrated in Fig. 8. The third harmonic must be terminated first since it has a shorter physical length. For example, an electrical short is implemented at node A using the $\lambda / 12$ stub at $3 f_{0}$, and then $\Gamma_{L . m}(3 \omega)$ is obtained from the short position using a transmission line with an appropriate electrical length $\theta_{7}$ and the characteristic impedance $Z_{m . O L}$. It should be noted that the magnitude of the implemented harmonic shorts may be less than unity due to lossy microstrip lines while the predicted ones are outside of the Smith chart, shown as

$$
\left|\Gamma_{L . \text { implemented }}(n \omega)\right| \leq\left|\Gamma_{L \cdot \text { predicted }}(n \omega)\right| .
$$

Therefore, performance degradation is expected due to the practical harmonic termination implementation. The third-harmonic termination affects the second $\Gamma_{L}(2 \omega)$ and fundamental $\Gamma_{L}(\omega)$ terminations. If the adjusted second-harmonic termination of the main amplifier is designated as $\Gamma_{L . m}^{\prime}(2 \omega)$, then it is terminated in a similar way using the $\lambda / 8$ open stub and the transmission line with the electrical length of $\theta_{8}$ and the characteristic impedance of $Z_{m . O L}$. The characteristic impedance $Z_{5}$ of the open stubs affects both the bandwidth and the fundamental loads $\Gamma_{L}(\omega)$. A narrower width exhibits smaller bandwidth but affects less $\Gamma_{L}(\omega)$ and vise versa. In this work for matching convenience a $100 \Omega$ impedance was used for reducing the impact on $\Gamma_{L}(\omega)$.

Those variations can be accurately calculated in simulation as shown in Fig. 9(a) in order to account for the practical fabrication parameters of the microstrip lines. The voltage waves injected into the matching circuit in Fig. 9(a) are

$$
\begin{aligned}
& V_{s 1 . m}=V_{1 . m . p}-50 \cdot I_{1 . m . p}, \\
& V_{s 1 . m}^{\prime}=V_{1 . m . i}+50 \cdot I_{1 . m . i}
\end{aligned}
$$

where $V_{1 . m . p}$ and $I_{1 . m . p}$ are the fundamental voltage and current predicted by the embedding network at the main amplifier output package reference plane. $V_{1 . m . i}$ and $I_{1 . m . i}$ are the fundamental voltage and current predicted at the reference plane before the impedance inverter. The direction of both currents $I_{1 . m . p}$ and $I_{1 . m . i}$ is flowing into the device drain node. The fundamental load variations at each reference plane are depicted in Fig. 9(b). The arrows indicate the direction where the loads provide high output power. $\Gamma_{L . m \text {.inv }}\left(\omega, P_{\text {out }}\right)$ are loads seen right before the output impedance inverter which are the intended loads after the harmonic matching. Note that the loads $\Gamma_{L . m}\left(\omega, P_{\text {out }}\right)$ and $\Gamma_{L . m . i n v}\left(\omega, P_{\text {out }}\right)$ in this figure are slightly different from the loads in Fig. 7(a) due to the difference between the models used. The extracted Angelov model was used in Fig. 7 while the manufacturer model (CREE Inc.) was used in Fig. 9. Also, the harmonic terminations in Fig. 7 are ideal.

The fundamental load variation versus swept power may no longer be a straight line when they are rotated back to the real axis of the Smith chart. Therefore, the characteristic 


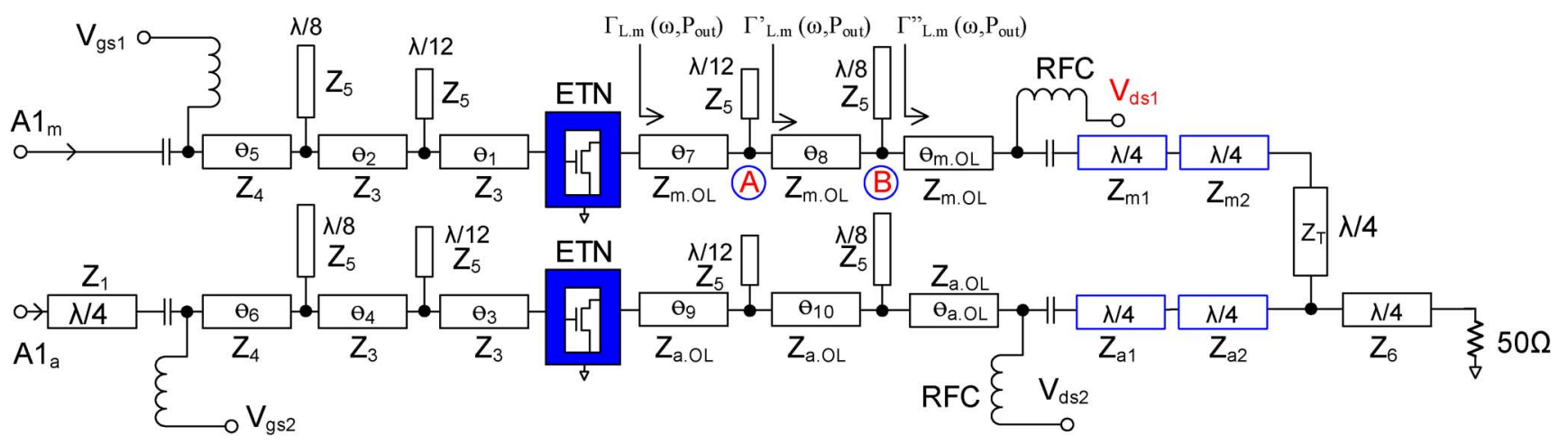

Fig. 8. Dual input asymmetric Doherty amplifier schematic.

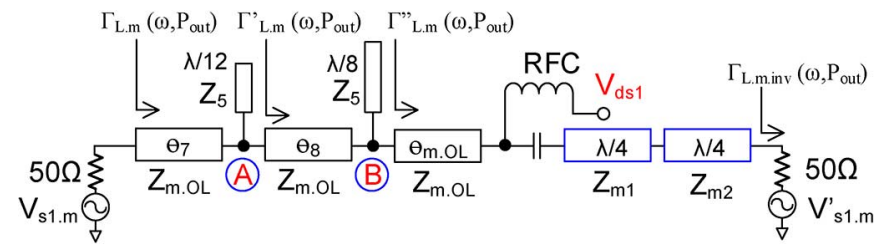

(a)

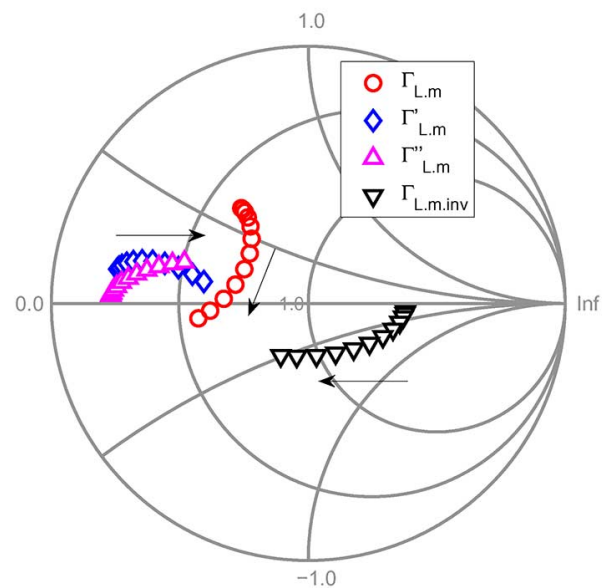

(b)

Fig. 9. (a) Each reference plane. (b) Fundamental load $\left(\Gamma_{L}\left(\omega, P_{\text {out }}\right)\right)$ variation versus power after the harmonic matching at each reference plane (a). The arrows indicate loads providing high output power.

impedance and the length of the offset line can be selected for the $\Gamma_{L}^{\prime \prime}\left(\omega, P_{\text {out.peak }}\right)$ and $\Gamma_{L}^{\prime \prime}\left(\omega, P_{\text {out.back }}\right)$ to best fit those loads, i.e., for the main amplifier

$$
\begin{aligned}
\Gamma_{L}^{\prime \prime}\left(\omega, P_{\text {out.peak }}\right) & =\frac{Z_{\text {in.peak }}-Z_{m . O L}}{Z_{\text {in.peak }}+Z_{m . O L}} \\
\Gamma_{L}^{\prime \prime}\left(\omega, P_{\text {out.back }}\right) & =\frac{Z_{\text {in.back }}-Z_{m . O L}}{Z_{\text {in.back }}+Z_{m . O L}}
\end{aligned}
$$

where

$$
\begin{aligned}
& Z_{\text {in.peak }}=Z_{m . O L} \frac{\alpha(n+1) R_{L}+j Z_{m . O L} \tan \theta_{m . O L}}{Z_{m . O L}+j \alpha(n+1) R_{L} \tan \theta_{m . O L}} \\
& Z_{\text {in.back }}=Z_{m . O L} \frac{\alpha(n+1)^{2} R_{L}+j Z_{m . O L} \tan \theta_{m . O L}}{Z_{m . O L}+j \alpha(n+1)^{2} R_{L} \tan \theta_{m . O L}} .
\end{aligned}
$$

An offset line of $19 \Omega$ with $154^{\circ}$ before the harmonic matching, was used in this work to rotate the fundamental loads at the package reference plane for it to lie on the real line before the load-side quarter-wave transformer. The same offset line was used for the auxiliary amplifier although it was fine tuned in the final design stage. The resulting load reflection coefficients measured after the offset lines for the main and auxiliary amplifiers are shown in Fig. 7 using cross symbols (black). It is noticed that the real loads have shifted to higher and lower values for the main and auxiliary amplifiers, respectively. This would indicate that to sustain the virtual intrinsic Doherty operation targeted at the current plane a modified Doherty transformer/combiner may be potentially needed at the external reference planes. However, in this particular design, the original Doherty transformer/combiner was found to still perform well due to the correlated resistive load variation of the main and auxiliary amplifiers.

\section{Load-Matching Network Bandwidth Analysis}

The fractional bandwidth of the proposed two-section impedance transformers can be analyzed and compared with the bandwidth of the Doherty impedance inverter. The fractional bandwidth for a single section quarter-wave transformer is [29]

$$
\frac{\Delta f}{f_{0}}=2-\frac{4}{\pi} \cos ^{-1}\left[\frac{\Gamma_{m}}{\sqrt{1-\Gamma_{m}^{2}}} \frac{2 \sqrt{Z_{0} Z_{L}}}{\left|Z_{L}-Z_{0}\right|}\right]
$$

where $\Gamma_{m}$ is the magnitude of the tolerable reflection coefficient.

Since the transformer converts $R_{L}$ to $(n+1)^{2} R_{L}$ at the backed-off power level, this can be rearranged as

$$
\frac{\Delta f}{f_{0}}=2-\frac{4}{\pi} \cos ^{-1}\left[\frac{\Gamma_{m}}{\sqrt{1-\Gamma_{m}^{2}}} \frac{2(n+1)}{n^{2}+2 n}\right] .
$$

In this work, $n=2$, and, if we assume a tolerable reflection coefficient $\Gamma_{m}=0.05$ for analysis purpose, the fractional bandwidth is $4.78 \%$. It should be noted that there is no bandwidth limitation of the impedance inverter at the peak power because the characteristic impedance of the inverter is the same as the modulated load at the peak power and also the same as $R_{\text {opt }}$ of the device. 


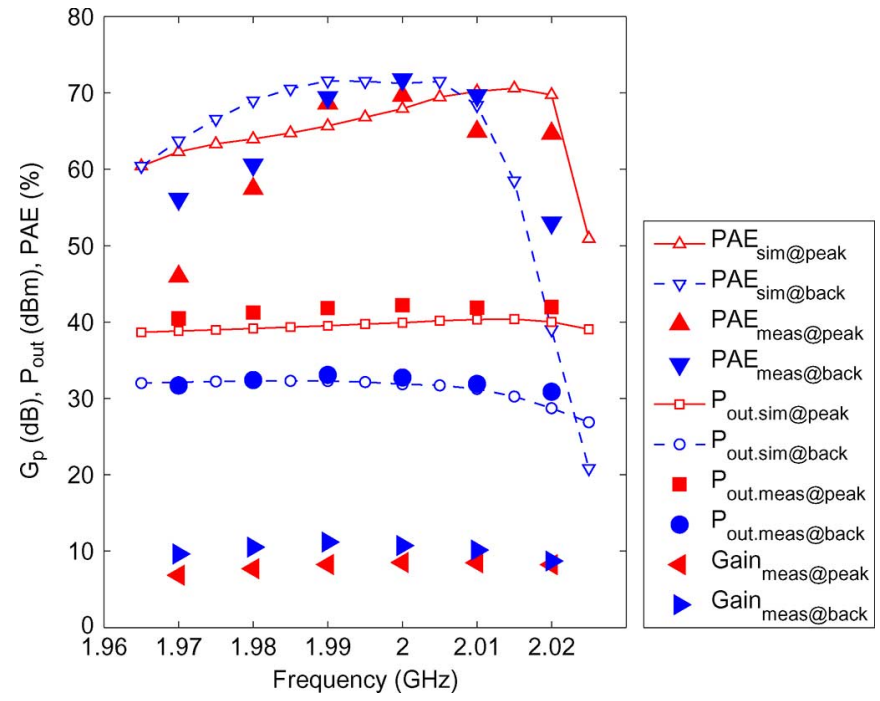

Fig. 10. Frequency dependence of the PAE, output power, and power gain.

The two-section quarter-wave transformer used in this work is a binomial transformer which fractional bandwidth be expressed as [29]

$$
\frac{\Delta f}{f_{0}}=2-\frac{4}{\pi} \cos ^{-1}\left[\frac{1}{2}\left(\frac{\Gamma_{m}}{|A|}\right)^{\frac{1}{N}}\right]
$$

where $N$ is the number of sections used and constant $A$ defined as

$$
A=2^{-N} \frac{Z_{L}-Z_{0}}{Z_{L}+Z_{0}}
$$

The impedance transform ratios for the main and auxiliary are 0.5 and 1.5 , respectively. Therefore, $A_{\text {main }}=1 / 12$ and $A_{\text {aux }}=$ $1 / 20$. Assuming the same $\Gamma_{m}=0.05$, the fractional bandwidth for the main and auxiliary amplifiers are $50.6 \%$ and $66.7 \%$, respectively. The transformer of the auxiliary amplifier shows wider bandwidth with less impedance transform ratio, as expected. According to the analysis, the two-section transformers have a much wider bandwidth than the Doherty impedance inverter, which provides the major bandwidth constraint in this load-matching network. Also, the harmonic short stubs are expected to have a bandwidth limitation similar to the single-section quarter-wave transformer inverter.

The simulated and measured bandwidth of the implemented Doherty amplifier in terms of gain, output power, and drain efficiency versus frequency is shown in Fig. 10. The output power, gain, and drain efficiency were measured for two different operating conditions. One is at the peak power operating condition and the other one is at the back-off power operating condition. The high performance of the PA ( $>50 \%$ PAE) is seen in Fig. 10 to be maintained for a bandwidth of $50 \mathrm{MHz}$. A reduced efficiency ( $>40 \%$ PAE) operation was verified to hold for a bandwidth in excess of $100 \mathrm{MHz}$. Note that these results do not reflect on the limitation of the nonlinear embedding PA design technique but rather on the particular design selected for the matching networks, inverter and offset lines. For example, the bandwidth improvement techniques proposed in [18]-[20]

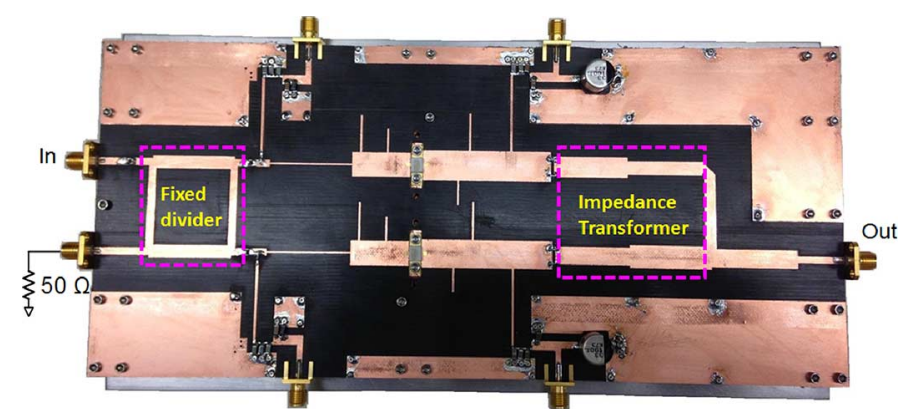

(a)

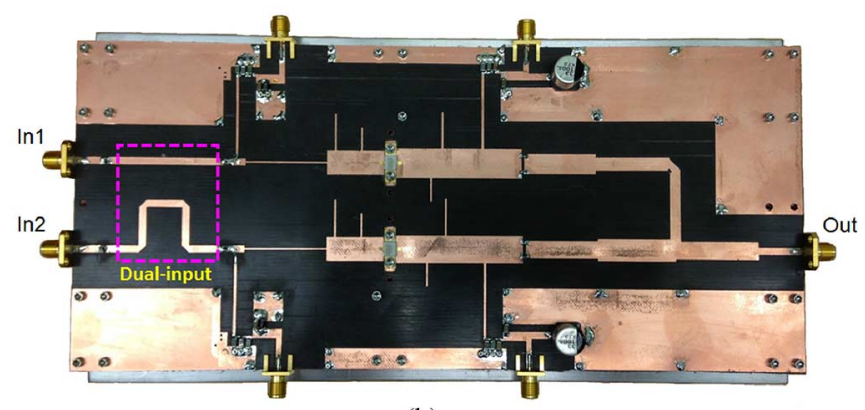

(b)

Fig. 11. (a) Fixed power-ratio divider Doherty PA from [5] and (b) the companion dual-input Doherty PA.

for the inverter could be implemented as needed for broadband design.

\section{SingLe-INPUT DOHERTY}

A single-input fixed-power-ratio-divider Doherty PA was built in [5] and shown in Fig. 11(a). The model used for the nonlinear embedding was replaced with the manufacturer (CREE Inc.) model at the final stage, and the design was fine-tuned using electromagnetic simulation results. More power was provided to the auxiliary amplifier to keep the peak current with the reduced gate voltage for class-C operation. The input coupler exhibited in simulation the following $S$-parameters $\left|S_{21}\right|=0.652$ and $\left|S_{31}\right|=0.749$, where 2 and 3 are the inputs of the main and auxiliary amplifiers, respectively. This yields an input power-division ratio of $1.2 \mathrm{~dB}$.

The power ratio $n=2$ between the main and auxiliary amplifiers is a design goal which is selected to obtain the intended back-off operation. This power ratio can be observed in the final stage nonlinear circuit simulation using the manufacturer model and electromagnetic simulation results as depicted in Fig. 12. As the input incident power is increased, the power ratio $n$ approaches the intended design goal of 2 .

The measurement results in Fig. 13 are repeated from [5] for readers' reference. The output power and drain currents for the main and auxiliary amplifiers were measured with swept input power at $2 \mathrm{GHz}$ using CWs. Three different auxiliary amplifier gate voltages $\left(V_{g s . a}=-3.4 \mathrm{~V},-3.6 \mathrm{~V}\right.$ and $\left.-3.8 \mathrm{~V}\right)$ were used to investigate the tradeoff between efficiency and linearity.

Fig. 13 compares the measured drain efficiency and power gain (symbols) to the simulated data (solid lines). It was observed that increasing the auxiliary gate voltage reduces the 


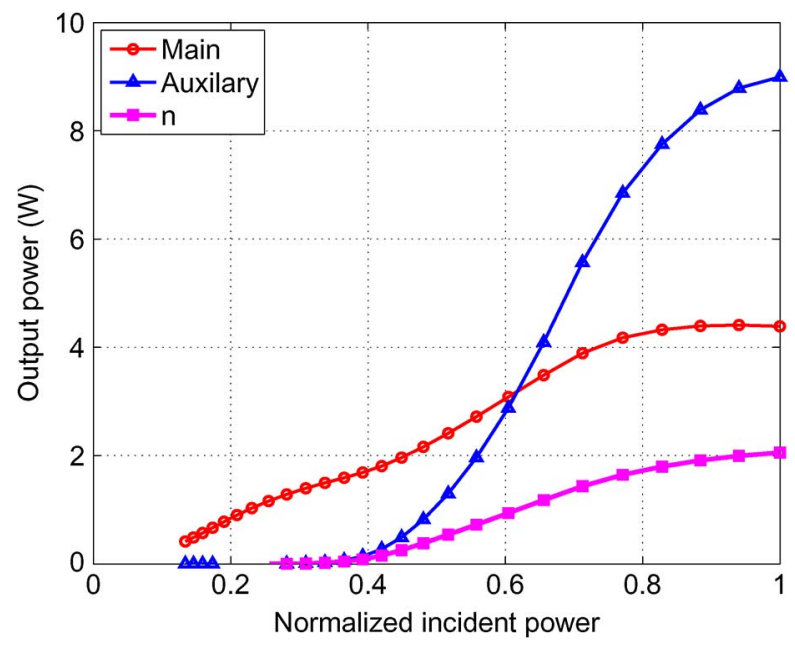

Fig. 12. Simulated output powers of both main and auxiliary amplifiers and power-division ratio $n$ are plotted versus normalized incident power.

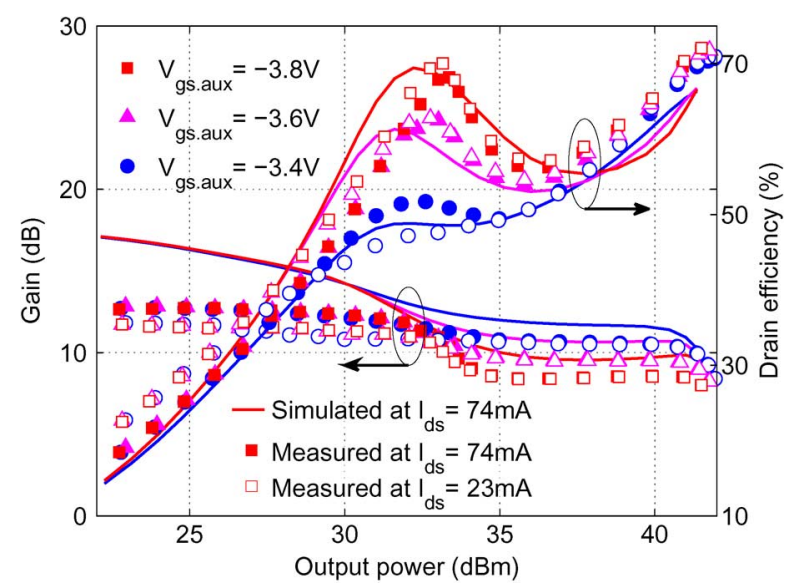

Fig. 13. Measured (solid and blank symbols) drain efficiency and gain at 2 $\mathrm{GHz}$ are compared with simulation (solid lines) with swept input powers at three different auxiliary amplifier gate voltages. Reduced main amplifier bias current from $74 \mathrm{~mA}$ (solid symbols) to $23 \mathrm{~mA}$ (blank symbols) shifts the efficiency curves to the left. A 74-mA drain current was used in the simulations. (Repeated from [5].)

second peak efficiency while flattening the gain curves. It is interesting to note that reducing the main amplifier quiescent current from $74 \mathrm{~mA}$ to $23 \mathrm{~mA}$ slightly shifts the efficiency curves to the left resulting in a wider back-off and flatter gain curves. Thus, the most flat gain was obtained at $-3.4 \mathrm{~V}$ for the auxiliary gate voltage and $23 \mathrm{~mA}$ for the main amplifier quiescent current but yielded reduced efficiency.

A lower auxiliary gate bias reduces the conduction angle of the class-C operation, and the device turn-on is retarded. The main amplifier is expected to be more saturated during this delayed turn-on and reaches higher efficiency level at the backed-off peak efficiency point. Since the main amplifier is saturated the gain is reduced as well and the auxiliary amplifier produces less power in this deeper class-C bias operation [15]. The measured drain efficiency at $-3.8 \mathrm{~V}$ of the auxiliary (square symbol, $\square$, red) is, therefore, the highest among the three biases and the gain is the lowest. As the auxiliary gate bias is increased, the conduction angle is increased in the class- $\mathrm{C}$ operation, and the device turn-on time is advanced. In summary,
TABLE I

AVERAGE EFFICIENCY BEFORE AND AFTER LINEARIZATION

\begin{tabular}{c|c|c|c|c}
\hline & $\begin{array}{c}\mathrm{P}_{\text {o.avg }} \\
(\mathrm{dBm})\end{array}$ & $\begin{array}{c}\mathrm{DE}_{a v g} \\
(\%)\end{array}$ & $\begin{array}{c}\text { ACPR } \\
(\mathrm{dBc})\end{array}$ & $\begin{array}{c}\text { NMSE } \\
(\mathrm{dB})\end{array}$ \\
\hline \hline Before DPD & 33.55 & 54.28 & -28.42 & -13.11 \\
\hline After DPD & 33.55 & 51.86 & -51.47 & -37.37 \\
\hline
\end{tabular}

the lowest gate bias $-3.8 \mathrm{~V}$, where the auxiliary amplifier turns on late, exhibited the highest efficiency with the lowest gain. On the contrary, the highest gate bias $-3.4 \mathrm{~V}$ where the auxiliary amplifier turns on early, exhibited the lowest efficiency with the highest gain. However, this experiment was conducted with a fixed power ratio divider at the input. When the gate bias of class- $\mathrm{C}$ operation is varied, the peak current is also expected to be varied requiring additional input power and different power division ratio. To investigate the impact of the auxiliary gate bias in more details we will characterize in the next section a dual input Doherty power amplifier built without the fixed power ratio divider as shown in Fig. 11(b). It is interesting to notice when the output power is between 30 and $35 \mathrm{dBm}$, the drain efficiency measured at $74 \mathrm{~mA}$ is slight lower than the one measured at $23 \mathrm{~mA}$ for both cases of $V_{\mathrm{gs} . a}=-3.8 \mathrm{~V}$ and $-3.6 \mathrm{~V}$. However, when $V_{\mathrm{gs} . a}=-3.4 \mathrm{~V}$, the drain efficiency with $74 \mathrm{~mA}$ is higher than the one with $23 \mathrm{~mA}$. Therefore further investigation would be necessary for the mechanism after the auxiliary device is turned on but the investigation of the mechanism at the transitional range is out of the scope of this paper.

It should be mentioned that the $\mathrm{CW}$ gain in Fig. 13 was calculated using the incident power instead of the input power to be consistent with the linearization measurement system used for modulated signal excitations. Above 50\% drain efficiency was maintained over a $11.4-\mathrm{dB}$ output power range at $-3.6 \mathrm{~V}$ for the auxiliary gate voltage and $23 \mathrm{~mA}$ for the main amplifier quiescent current depicted by magenta blank triangles in Fig. 13. A drain efficiency of $71 \%$ at the peak power of $41.8 \mathrm{dBm}$ and $62.7 \%$ at the second peak of $32.8 \mathrm{dBm}$ (9 $\mathrm{dB}$ back-off) were observed. For these operating conditions, the digital predistortion (DPD) from [22] using a field-programmable gate array (FPGA) test-bed in [23] was applied for demonstration purposes. A 10-MHz-bandwidth LTE signal with 8.85-dB PAPR was used. The same average output power of $33.55 \mathrm{dBm}$ was used before and after applying DPD for a fair comparison. Thus, in this DPD experiment, using modulated signals, the $\mathrm{PA}$ reaches a peak power of $42.4 \mathrm{dBm}(16.7 \mathrm{~W})$ which is $0.6 \mathrm{~dB}$ above the measured $41.8-\mathrm{dBm}$ peak power using $\mathrm{CW}$ signals. An average efficiency of $51.86 \%$ was observed after linearization maintaining -51.47-dBc ACPR and -37.37-dB normalized mean-square error (NMSE). The nonlinearity order was 15 and the memory depth 7 . The measured linearization results are summarized in Table I and illustrated in Fig. 14.

\section{DUAL-INPUT DOHERTY}

Here, a dual-input Doherty PA without a fixed power-ratio divider is built and measured to investigate the impact of the auxiliary gate bias. A systematic large-signal characterization method for the dual-input Doherty is presented. An algorithm 


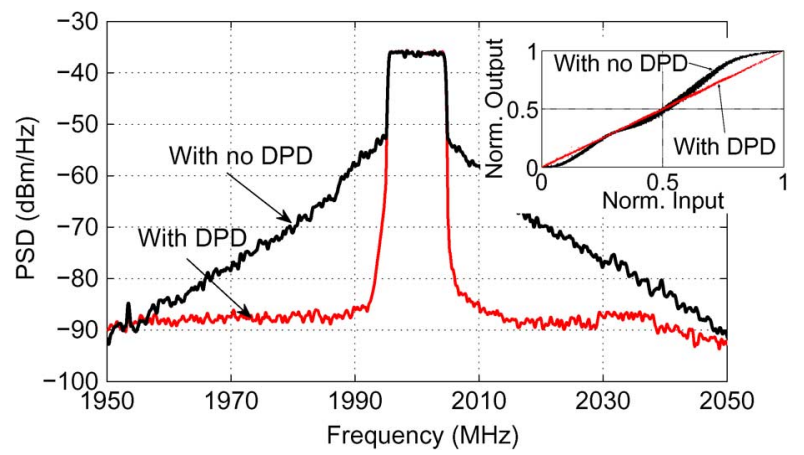

Fig. 14. Spectrum of normalized output and AM-AM distortion were measured at $-3.6 \mathrm{~V}$ for the auxiliary gate voltage and $23 \mathrm{~mA}$ main amplifier bias current. PSD stands for power spectral density.

to determine the constant-gain maximum-efficiency conditions from the systematic characterization data is also proposed. Simulation and measured data are then discussed and compared with the single-input fixed-power ratio-divider Doherty measurements.

\section{A. Constant-Gain Maximum-Efficiency Algorithm}

An algorithm to determine the input conditions using the additional degrees of freedom of the amplitude and phase of the auxiliary amplifier is introduced. This algorithm selects the best efficiency while maintaining a constant power gain in the highpower Doherty load-modulation range. The three independent variables of the $\left|A 1_{m}\right|,\left|A 1_{a}\right|$ and $\angle A 1_{a}$ associated with the main $A 1_{m}$ and auxiliary $A 1_{a}$ incident waves are systematically swept. It should be mentioned that the auxiliary amplifier is off in the low-power region ignoring soft turn-on. The magnitude and phase of the auxiliary amplifier can be normalized relative to the main amplifier input according to

$$
\begin{aligned}
\Delta P_{a} & =10 \log \left(\frac{\left|A 1_{a}\right|}{\left|A 1_{m}\right|}\right)^{2} \\
\Delta \angle A 1_{a} & =\angle\left(\frac{A 1_{a}}{A 1_{m}}\right) .
\end{aligned}
$$

The reference gain for the dual-input Doherty PA is defined for convenience as the ratio between the Doherty output power $P_{\text {out }}$, and the main amplifier incident power, $P_{\text {inc. } m}$. The gain surface can be plotted in the $\Delta P_{a}$ and $\Delta \angle A 1_{a}$ space at each incident main amplifier power level, as shown in Fig. 15(a). A set of input conditions $\left(\left\{\left(\Delta P_{a . k}, \angle A 1_{a . k}\right)\right\}, k=1, \cdots, K\right)$ for the auxiliary amplifier can be selected for a given constant gain, as illustrated by the solid black line in this 2-D plot. $K$ is the number of points used. The drain efficiency can then be calculated at these points as shown by the solid black line in Fig. 15(b) on the efficiency surface generated at the same incident main amplifier power level in the same $\Delta P_{a}$ and $\Delta \angle A 1_{a}$ space. Finally, the input condition giving the maximum drain efficiency can be selected along the line. A set of input conditions $\left(\left\{\left(\Delta P_{a . m}, \angle A 1_{a . m}\right)\right\}, m=1, \cdots, M\right)$ can be selected at

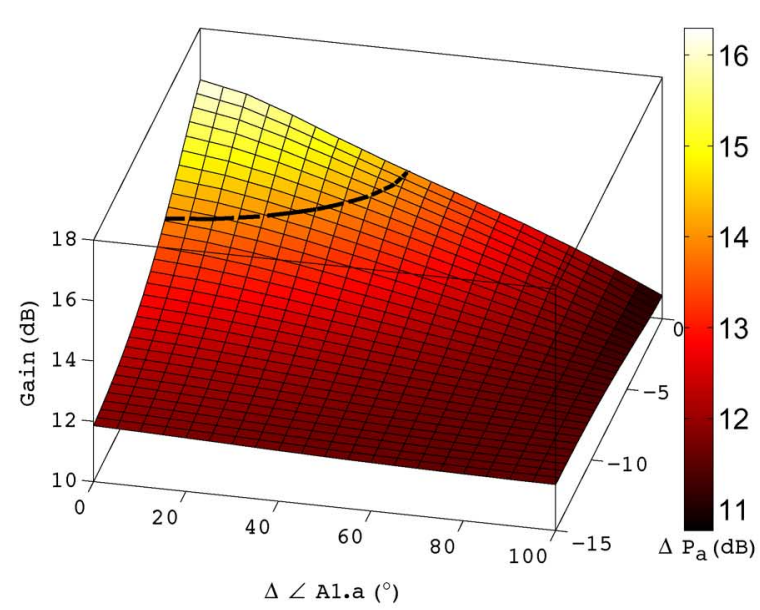

(a)

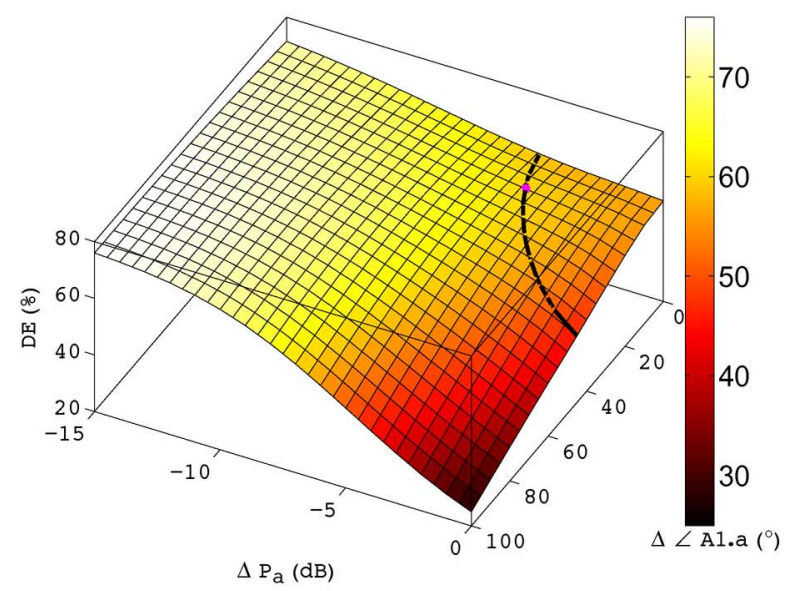

(b)

Fig. 15. (a) Simulated gain and (b) drain efficiency surfaces at the main amplifier at an incident power of $22.1 \mathrm{dBm}$ at $2 \mathrm{GHz}$. The black line corresponds to a 14-dB constant gain locus. The red dot corresponds to the maximum efficiency along the constant gain locus.

each incident main amplifier power level. $M$ is the number of incident main amplifier power levels.

\section{B. Simulation Results}

The dual-input Doherty amplifier was simulated in Agilent ADS using the CREE CGH27015F model at $2 \mathrm{GHz}$ with CWs. The passive networks were simulated using the Momentum simulation and the simulated data was used for the amplifier circuit simulations. The incident main amplifier power was swept from 17.1 to $28.1 \mathrm{dBm}$. The relative incident power of the auxiliary amplifier $\Delta P_{a}$ was swept from -15 to $0 \mathrm{dBc}$. The relative phase of the auxiliary amplifier $\Delta \angle A 1_{a}$ was swept from $0^{\circ}$ to $100^{\circ}$. It should be noted that the simulation results showed symmetry around $0^{\circ}$. Therefore, only one side (from $0^{\circ}$ to $100^{\circ}$ ) was selected for applying the algorithm to avoid multiple maximums.

Fig. 16 shows the simulated drain efficiency versus output power at $V_{\text {gs. } a}=-3.4 \mathrm{~V}$ for all of the systematically swept input conditions. The fixed power-divider Doherty amplifier simulation (solid line, black) and measured (square symbol, $\square$, black) results are plotted for references. The CGME algorithm was applied to the simulated data and the selected points by 


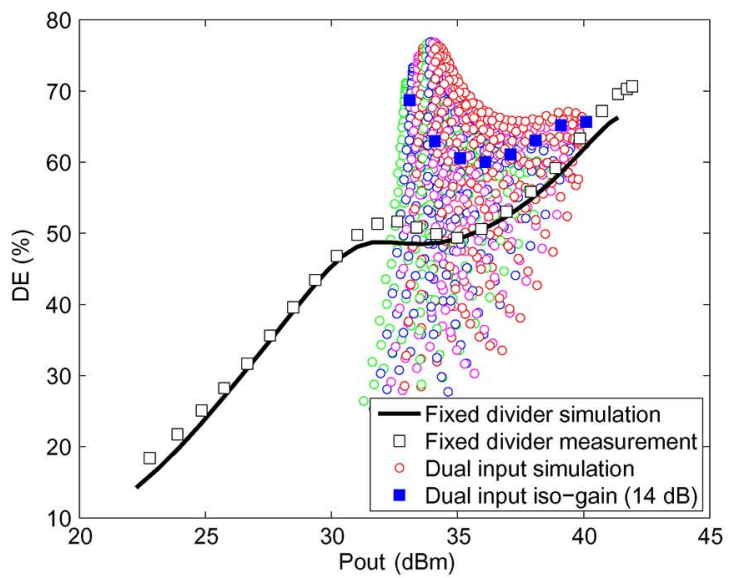

(a)

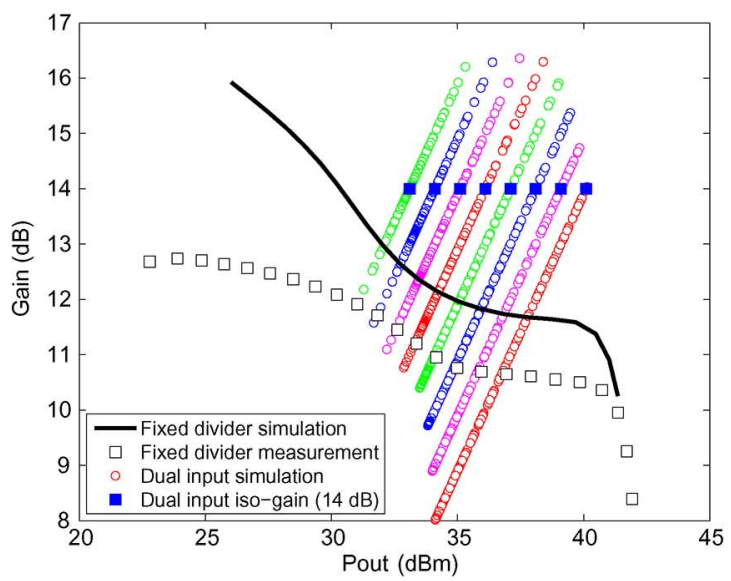

(b)

Fig. 16. Simulated (a) drain efficiencies and (b) gain for all of the systematically swept input conditions at $V_{\mathrm{gs} . a}=-3.4 \mathrm{~V}$ were compared with fixed power-divider Doherty and the constant gain algorithm.

the algorithm are shown with square symbols (blue) in the figure. The simulated results show that there is a possibility that the drain efficiency can be further improved by controlling the main and auxiliary inputs separately even keeping constant gain. It should be noted that AM-to-PM nonlinearity is not considered in the CGME algorithm.

\section{Measurement Setup}

The dual-input Doherty power amplifier was fabricated as shown in Fig. 11(b) with the fixed power divider shown in Fig. 11(a) removed. A $90^{\circ}$ delay line was added to the auxiliary input. Continuous waveform was performed at $2 \mathrm{GHz}$ using a large-signal network analyzer (LSNA, MT4463A) configured as shown in Fig. 17. Since the amplifier has two inputs and a single output, the two calibrated LSNA input and output ports were used for the dual inputs. Another coupler connected with an attenuator was calibrated using the LSNA and connected between the output port of the amplifier and a power meter (HP E4418). The two signal sources used at the input (Agilent ESG 4438C, MXG N5183A) were synchronized sharing a $10 \mathrm{MHz}$ reference signals. The auxiliary input amplitude and phase were automatically synchronized to the input of the main amplifier before each parameter-sweep to avoid any amplitude and phase drift during the measurement sweep. The amplitude

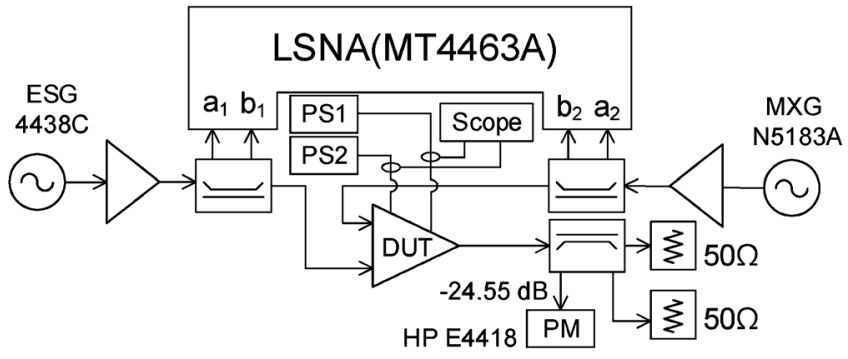

Fig. 17. Dual-input Doherty PA characterization setup.

$\left(\Delta P_{a}\right)$ and phase $\left(\Delta \angle A 1_{a}\right)$ of the auxiliary amplifier were varied to the intended values right after the synchronization. The three inputs were systematically swept, the incident main amplifier power $P_{\text {inc. } m}$ varying from 18.2 to $28.2 \mathrm{dBm}$, the relative auxiliary incident power $\Delta P_{a}$ from -9 to $1 \mathrm{dBc}$, and the relative auxiliary phase $\Delta \angle A 1_{a}$ from $-70^{\circ}$ to $20^{\circ}$.

The main amplifier was biased at $14.3 \mathrm{~V}, 74 \mathrm{~mA}$ for the drain voltage and currents, respectively. The auxiliary amplifier was biased at $28 \mathrm{~V}$ for the drain voltage. The measurements were performed at three different auxiliary gate voltages of -3.4 , -3.6 , and $-3.8 \mathrm{~V}$.

\section{Measured Results and Discussion}

The measured drain efficiencies at $V_{\text {gs.a }}=-3.4 \mathrm{~V}$ are plotted in Fig. 18 for all the swept dual-input conditions. The fixed-power-divider Doherty amplifier simulation (solid line, black) and measurement (square symbol $\square$, black) are repeated for references. The CGME algorithms were applied to the measured data and shown with solid squares (blue). The simulated CGME result is also shown as a dashed line (black) for a reference. The $\mathrm{CW}$ measured results also predicts the possibility of an efficiency improvement by dynamically controlling the dual-input Doherty amplifier while maintaining a constant gain. As a result, simultaneous efficiency and linearity improvements are expected for such dual-input operation.

The optimal offset power and phase $\left(\Delta P_{a}, \Delta \angle A 1 . a\right)$ for the maximum drain efficiency are plotted in Fig. 19 for the three different auxiliary bias conditions $\left(V_{\text {gs. } .}=-3.4,-3.6\right.$, and $-3.8 \mathrm{~V}$ ) using three different constant gain values of $12.5,11.8$, and $11.4 \mathrm{~dB}$, respectively. The achievable constant gain is determined by the auxiliary gate bias. Also, the measured data fluctuation affects the achievable constant gain in the application of CGME algorithm. Therefore, the constant gains were manually selected by observing the measured data. The relative power offset yielding the optimal drain efficient is seen to vary with the output power. The phase inputs exhibit a smaller variation centered around $-30^{\circ}$ as the output power varies. The CGME efficiency results for the three cases are also plotted in Fig. 20. To test the sensitivity of the efficiency to the offset phase, the offset phase was held constant at $-30^{\circ}$ at its optimum value, and only the amplitude was varied (not shown here). In this experiment, the same level of efficiency was obtained, confirming that the relative insensitivity of the CGME efficiency to the offset phase for this particular Doherty amplifier.

The slope of the offset power versus output power curves in Fig. 19(a) implies that a nonlinear control on the auxiliary input 


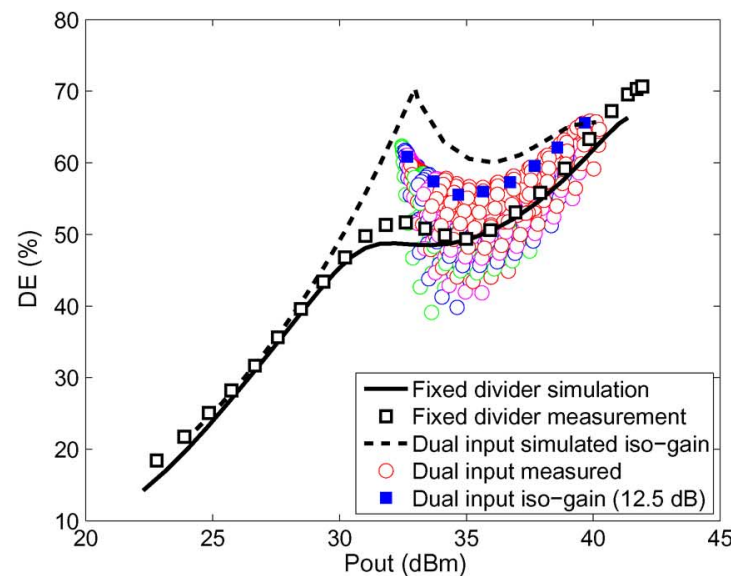

(a)

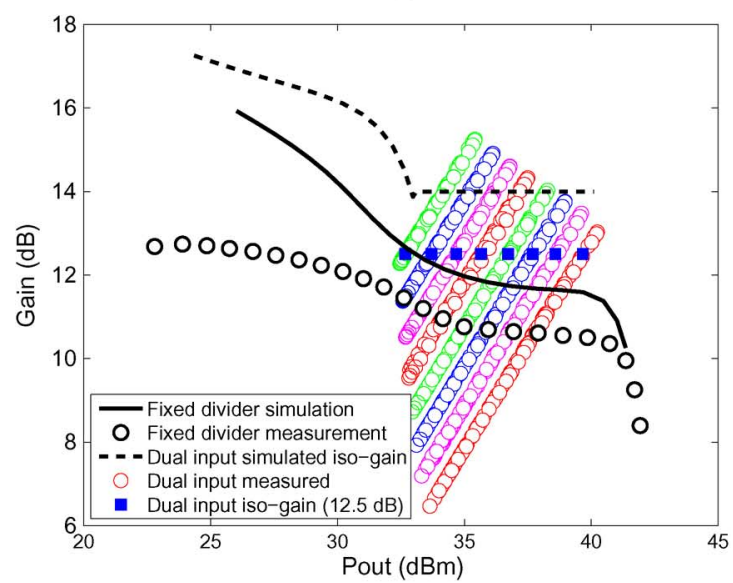

(b)

Fig. 18. Measured (a) drain efficiency and (b) gain using CW signals for the three input variables swept at $V_{\mathrm{gs} . a}=-3.4 \mathrm{~V}$.

power relative to the main input power is necessary to achieve the optimal constant-gain and maximum-efficiency operation condition. As can be seen, for the three gate-bias cases shown, the higher the auxiliary gate bias (earlier turn-on), the larger the slope and the requirement for a nonlinear control for the auxiliary input power. At a lower gate voltage $(-3.8 \mathrm{~V})$ with a slower turn-on, a flatter slope was observed resulting in a larger power offset. An approximate fixed unequal power divider could then be used, thus avoiding the need for the nonlinear control of the auxiliary input power. This results indeed justifies the improved efficiency performance achieved by the single-input Doherty $\mathrm{PA}$ for the $-3.8-\mathrm{V}$ gate bias as shown in Fig. 13. Note that, in the single-input Doherty PA, a fixed unequal power divider with 1.2-dB power division was used which is represented in Fig. 19(a) using a dashed line. This dashed line is seen to be well centered on the $-3.8-\mathrm{V}$ optimal offset line (red line) predicted by the CGME algorithm. To bring theoretical support to this experimental results, it is demonstrated in Appendix II that, for the case of an ideal transistor with constant transconductance and abrupt cutoff at the threshold voltage, there exists a unique optimal coupler ratio $k_{\max }$ for which the required Doherty operation is achieved at peak power and at back-off when using a class- $\mathrm{C}$ amplifier for the auxiliary amplifier.

The above characterization using $\mathrm{CW}$ implies that, if a fixed bias is used without dual-input control, $V_{\text {gs. } a}=-3.8 \mathrm{~V}$ should

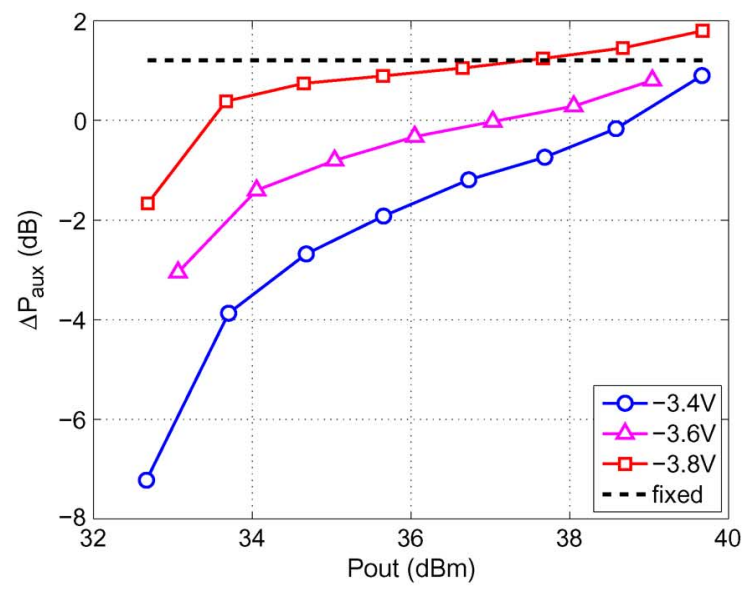

(a)

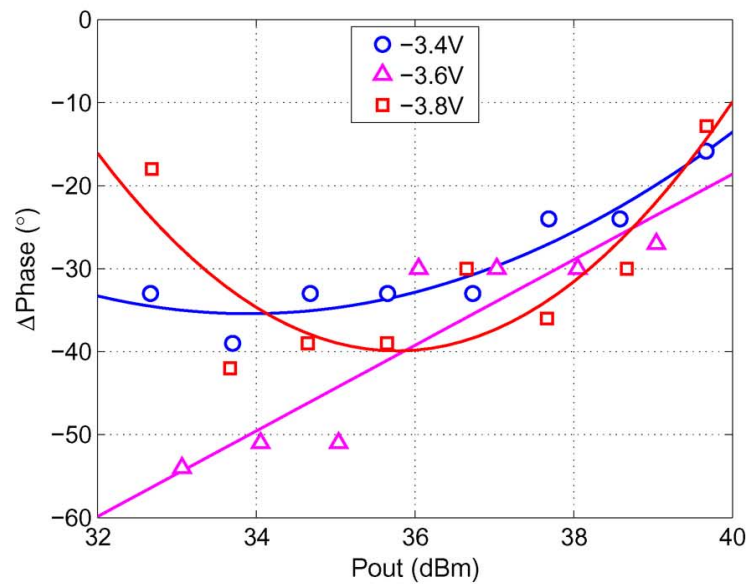

(b)

Fig. 19. Relative (a) power and (b) phase yielding an optimal efficiency for three different gate biases. These results are extracted from the measured data using the CGME algorithm for a constant gain of $12.5,11.8$, and $11.4 \mathrm{~dB}$ at the gate biases of $-3.4,-3.6$, and $-3.8 \mathrm{~V}$, respectively.

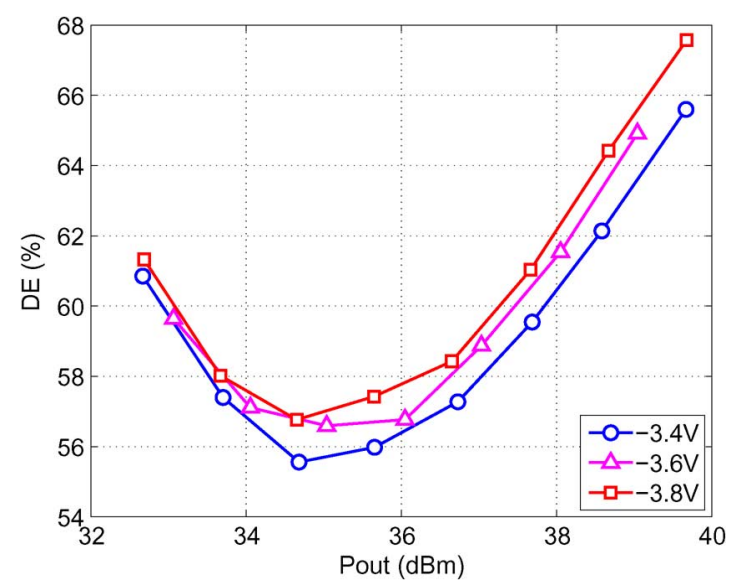

Fig. 20. Optimal drain efficiencies obtained for the three auxiliary gate biases $\left(V_{\mathrm{gs} . a}=-3.4,-3.6\right.$, and $\left.-3.8 \mathrm{~V}\right)$ using the proposed CGME algorithm.

give the highest efficiency over the other two cases which require nonlinear dual-input control. To validate the efficiency performance predicted above, the average efficiency was measured for a single-carrier WCDMA signal with 9.75-dB PAPR with the PA biased with $I_{\text {DS. } m}=23 \mathrm{~mA}, V_{\text {DS. } m}=14.3 \mathrm{~V}$, 
TABLE II

Average Drain EFFICIENCY, ACPR, AND NMSE Versus the AuXILIARY pa Gate Bias With a Constant Power-Division Ratio. The Presented VAlues ARE AFter DPD was APPLIED. $\triangle$ Phase $=0^{\circ}$

\begin{tabular}{c|c|c|c|c|c|c}
\hline \begin{tabular}{c}
$\mathrm{V}_{\text {gs.a }}(\mathrm{V})$ \\
\hline-3.8
\end{tabular} & $\begin{array}{c}\mathrm{P}_{\text {out.avg }}(\mathrm{W}) \\
1.01\end{array}$ & $\begin{array}{c}\Delta \mathrm{P}_{\text {aux }} \\
(\mathrm{dBc})\end{array}$ & $\begin{array}{c}\mathrm{ACPR}_{l} \\
(\mathrm{dBc})\end{array}$ & $\begin{array}{c}\mathrm{ACPR}_{h} \\
(\mathrm{dBc})\end{array}$ & $\begin{array}{c}\mathrm{NMSE} \\
(\mathrm{dB})\end{array}$ & $\begin{array}{c}\mathrm{DE} \\
(\%)\end{array}$ \\
\hline-3.6 & 1.00 & 1.45 & -44.3 & -45.4 & -31.0 & 57.9 \\
\hline-3.4 & 1.00 & 1.47 & -59.5 & -50.1 & -37.8 & 54.6 \\
\hline
\end{tabular}

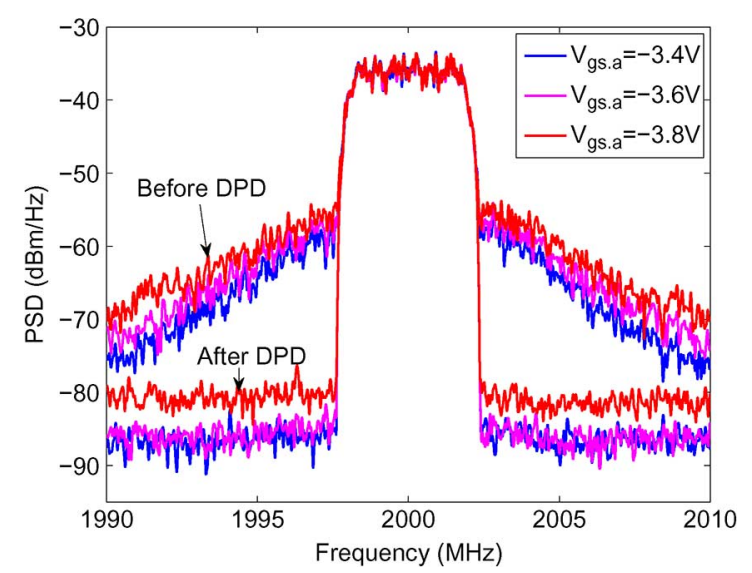

Fig. 21. Spectrum of normalized outputs before and after the DPD with the three different auxiliary gate voltages.

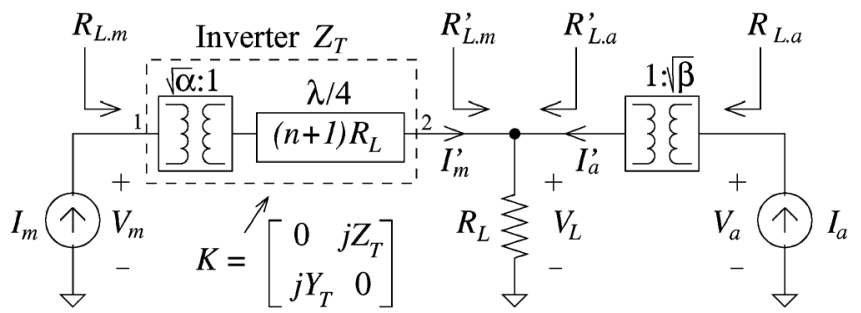

Fig. 22. Generalized Doherty circuits using two transformers with turn ratios $\alpha$ and $\beta$, respectively.

$V_{\text {DS } . a}=28 \mathrm{~V}$ using the same three gate-bias voltages. The experiment results are summarized in Table II. The fixed power ratio $\Delta P_{\text {aux }}$ was manually set to be approximately equal to $1.2 \mathrm{~dB}$. The phase difference $\Delta$ Phase was set to $0^{\circ}$ for experimental convenience. A DPD linearization was performed using a nonlinear order of 15 and memory length of 7 while keeping the average output power at $1.0 \mathrm{~W}$. As expected, it is shown that, under the same condition, the lower the gate voltage is, the higher the efficiency is at the cost of a slightly degraded NMSE due to the increased PA nonlinearity. An average efficiency of $57.9 \%$ was obtained at $-3.8 \mathrm{~V}$. The spectrum of normalized outputs before and after the DPD with the three different auxiliary gate voltages are shown in Fig. 21.

\section{CONCLUSION}

A novel design procedure for asymmetric Doherty PA using a model-based nonlinear embedding technique has been introduced and demonstrated. The nonlinear embedding technique accurately synthesizes the required waveforms at the external reference planes given the desired intrinsic Doherty operation targeted. This technique can significantly simplify the design process by removing the need for extensive harmonic-source/load-pull measurements or simulations. The proposed design procedure demonstrates the use of the nonlinear embedding technique when the intrinsic circuit operation involves the interaction between multiple active devices. A matching-network technique allowing for the implementation of an asymmetric Doherty PA using the same device is proposed. Finally, a methodology for simultaneously projecting the intrinsic waveforms of both devices to the package reference planes using the nonlinear embedding and designing the compensation networks is demonstrated.

Using the above procedure, a 9-dB back-off asymmetric Doherty amplifier has been designed. An even-numbered multisection impedance transformers and a reduced drain voltage of the main amplifier are also used to implement the asymmetric Doherty operation for the case where identical devices are used. A bandwidth analysis of the design demonstrated that the primary bandwidth limitation of the Doherty amplifier is limited by the quarter-wavelength impedance inverter given the added multisection impedance transformers exhibit a wider bandwidth.

Asymmetric Doherty operation was investigated with various auxiliary amplifier gate-bias voltages. A single-input Doherty amplifier with a fixed power-ratio divider was compared with a dual-input Doherty amplifier without a fixed power-ratio divider. A systematic dual-input Doherty amplifier characterization method was proposed, and a constant-gain maximum-efficiency algorithm was proposed for optimizing the input power division between the main and auxiliary amplifiers. The optimal performance obtained with the dual-input Doherty PA for the various gate voltages were found to be consistent with the results obtained for the single-input Doherty PA. These measurements points also toward the potential benefit in using nonlinear power division to maximize the efficiency while maintaining a constant gain. The PA performance obtained with the $\mathrm{CW}$ signals for the various auxiliary gate biases were further verified using WCDMA waveforms in terms of average efficiency and NMSE for the constant input power-division case.

\section{APPENDIX I}

Consider the generalized asymmetric Doherty circuit in Fig. 22. The matrix $K$ represents the ABCD matrix of the $Z_{T}$ inverter between ports 1 and 2 . The following generalized Doherty equations for $I_{m}$ and $I_{a}^{\prime}$ accounts for the transformer turn ratios $\alpha$ and $\beta$ introduced in this paper:

$$
\begin{aligned}
I_{m} & =\frac{1}{2} g_{m \cdot m} V_{\mathrm{GS} \cdot m} \\
I_{a}^{\prime} & =-j \sqrt{\alpha}(n+1)\left[I_{m}-\frac{I_{m \cdot \max }}{n+1}\right]=\sqrt{\beta} I_{a} .
\end{aligned}
$$

A factor $1 / 2$ for $I_{m}$ arises assuming the main operates in class B. It is also assumed that the main phase is selected such that $I_{m}=\left|I_{m}\right|$ and $V_{\mathrm{GS} . m}=\left|V_{\mathrm{GS} . m}\right|$. Note that the maximum main RF drain current $I_{a \text {. max }}$ at the fundamental frequency verifies $I_{a \cdot \max }=I_{\max . a} / 2$ where $I_{\text {max.a }}$ is the peak drain current sustained in class $\mathrm{F} / \mathrm{B}$ operation. With the use of the additional 
weighting factor $\sqrt{\alpha}$, one can easily verify that the main voltage $V_{m}$ verifies (using $Y_{T}=1 / Z_{T}$ )

$$
\begin{aligned}
V_{m} & =\frac{I_{m} Z_{T}^{2}}{R_{L}}+\frac{I_{a}^{\prime}}{j Y_{T}} \\
& =g_{m . m} V_{\mathrm{GS} . m}\left[\frac{Z_{T}^{2}}{R_{L}}-\frac{\sqrt{\alpha}(n+1)}{Y_{T}}\right]+\sqrt{\alpha} Z_{T} I_{m \cdot \max } .
\end{aligned}
$$

To obtain a constant voltage $V_{m}$ at the main transistor, the inverter impedance must be selected to be

$$
Z_{T}=\sqrt{\alpha}(n+1) R_{L}
$$

This in turn yields a modified constant main RF voltage $V_{m}$ defined as

$$
\begin{aligned}
V_{m . \max } & =V_{m}=\sqrt{\alpha} Z_{T} I_{m \cdot \max } \\
& =\alpha(n+1) R_{L} I_{m \cdot \max } \\
& =\sqrt{\frac{\alpha}{\beta}}\left|V_{a \cdot \max }\right| .
\end{aligned}
$$

The Doherty operation then imposes the following design equation expressed in terms of the voltage and current ratios $\gamma_{V}$ and $\gamma_{I}$ at the transistor current sources:

$$
\gamma_{V}=\frac{\left|V_{m \cdot \max }\right|}{\left|V_{a \cdot \max }\right|}=\sqrt{\frac{\alpha}{\beta}} \quad \gamma_{I}=\frac{\left|I_{m \cdot \max }\right|}{\left|I_{a \cdot \max }\right|}=\frac{1}{n} \sqrt{\frac{\beta}{\alpha}}
$$

where $n$ is the maximum auxiliary-to-main PA power-ratio delivered:

$$
n=\frac{P_{\mathrm{RF} . a}}{P_{\mathrm{RF} \cdot m}}=\frac{\mathcal{R}\left\{V_{a \cdot \max } I_{a \cdot \max }^{*}\right\}}{\mathcal{R}\left\{V_{m \cdot \max } I_{m \cdot \max }^{*}\right\}}=\frac{1}{\gamma_{V} \gamma_{I}} .
$$

It becomes evident that a continuum of Doherty solutions in terms of maximum voltage and current ratios $\gamma_{V}$ and $\gamma_{I}$ is possible for a given targeted power ratio $n$ to satisfy $n=1 /\left(\gamma_{V} \gamma_{I}\right)$. For example, for $n=2,\left(\gamma_{V}=1, \gamma_{I}=.5\right)$ and $\left(\gamma_{V}=\right.$ $\left..5, \gamma_{I}=1\right)$ are two possible solutions. The latter one is adopted in this paper, whereas the former is the classic asymmetric design [3]. Further, for the voltage ratio $\gamma_{V}$ selected, we have the design equation $\alpha / \beta=\gamma_{V}^{2}$. Again, a continuum of design solutions with $\alpha=\gamma_{V}^{2} \beta$ is then possible. The impedances range reported in Section II for this generalized design then provides a design guide to achieve the preferred load ranges for the main and auxiliary transistors. The generalized asymmetric Doherty design equations reduce to the classic asymmetric Doherty design equations [3] when selecting $\gamma_{V}=\alpha=1$, which leads to $\beta=1$ and $\gamma_{I}=1 / n$.

\section{APPENDIX II}

Here, we derive the design equation for the selection of the optimal coupler coefficient $k_{\max }$. According to the generalized
Doherty theory presented in Appendix I, the RF fundamental current of the auxiliary transistor should be of the form (assuming $I_{m}=\left|I_{m}\right|$ )

$$
I_{a}=-j(n+1) \sqrt{\frac{\alpha}{\beta}}\left[I_{m}-\frac{I_{m \cdot \max }}{n+1}\right]
$$

such that we have $\left|I_{a \cdot \max }\right|=n \sqrt{\alpha / \beta} \cdot I_{m \cdot \max }$.

Let us assume that the main transistor exhibits the following linear transfer characteristics when operating in class B:

$$
I_{m}=\frac{1}{2} g_{m . m} V_{\mathrm{GS} . m}
$$

with $V_{\mathrm{GS} . m}=\left|V_{\mathrm{GS} . m}\right|$. This results in the required auxiliary current being expressed as

$$
I_{a}=-j\left[\frac{n+1}{2} \sqrt{\frac{\alpha}{\beta}} g_{m . m}\left|V_{\mathrm{GS} . m}\right|-\sqrt{\frac{\alpha}{\beta}} I_{m \cdot \max }\right] .
$$

The auxiliary PA is implemented with a transistor operating in class $\mathrm{C}$ which ideally yields the RF fundamental current (assuming $\left.V_{\mathrm{GS} . a}=-j\left|V_{\mathrm{GS} . a}\right|\right)$

$$
I_{a}=-j W(\theta) g_{m . a}\left(\left|V_{\mathrm{GS} . a}\right|+V_{\mathrm{GS} . d c . a}-V_{T}\right)
$$

where $V_{\mathrm{GS} . d c . a}$ is the dc gate bias for the auxiliary transistor and $W(\theta)$ is a weighting factor dependent on the conduction angle $\theta$. $W$ is given by [24]

$$
W(\theta)=\frac{1}{2 \pi} \frac{\theta-\sin \theta}{1-\cos \left(\frac{\theta}{2}\right)}
$$

where $\theta$ the class-C conduction angle defined as

$$
\cos \left(\frac{\theta}{2}\right)=\frac{V_{T}-V_{\mathrm{GS} . d c . a}}{\left|V_{\mathrm{GS} . a}\right|} .
$$

For large $\left|V_{\mathrm{GS} . a}\right|$, the conduction angle approaches $\theta=\pi$ of the class-B operation, and the weighting factor $W(\theta)$ converges toward $1 / 2$ like in (24).

Equating the required Doherty current (25) with the class-C current provided in (26), we obtain the required auxiliary RF gate voltage amplitude $\left|V_{\mathrm{GS} . a}\right|$ in terms of the main RF gate voltage $V_{\mathrm{GS} . m}$

$$
\begin{aligned}
\left|V_{\mathrm{GS} . a}\right|=\frac{n+1}{2 W(\theta)} \sqrt{\frac{\alpha}{\beta}} \frac{g_{m . m}}{g_{m \cdot a}}\left|V_{\mathrm{GS} . m}\right| \\
-\sqrt{\frac{\alpha}{\beta}} \frac{I_{m \cdot \max }}{W(\theta) g_{m . a}}+V_{T}-V_{\mathrm{GS} . d c . a} .
\end{aligned}
$$

We shall select the de gate bias voltage $V_{\mathrm{GS} . d c . a}$ such that

$$
V_{T}-V_{\mathrm{GS} . d c . a}=\sqrt{\frac{\alpha}{\beta}} \frac{I_{m . \max }}{W\left(\theta_{\max }\right) g_{m . a}}
$$


where $\theta_{\max }$ is the conduction angle at which the auxiliary current reaches its peak current $I_{a \text {.max }}$. We can then easily verify that the two edge boundary conditions are satisfied as follows:

$$
\begin{aligned}
\left|V_{\mathrm{GS} . a}(\theta=0)\right| & =k_{\max }\left|V_{\mathrm{GS} . m}(\theta=0)\right| \\
& =k_{\max } \frac{2}{g_{m . m}} \frac{I_{m . \max }}{n+1} \\
& =V_{T}-V_{\mathrm{GS} . d c . a} \Rightarrow I_{a}(\theta=0) \\
& =0 \\
\left|V_{\text {GS.a }}\left(\theta_{\max }\right)\right| & =k_{\max }\left|V_{\mathrm{GS} . m}\left(\theta_{\max }\right)\right| \Rightarrow I_{a}\left(\theta_{\max }\right) \\
& =I_{a . \max },
\end{aligned}
$$

with the coupler ratio $k_{\max }$ given by

$$
k_{\max }=\frac{n+1}{2 W\left(\theta_{\max }\right)} \sqrt{\frac{\alpha}{\beta}} \frac{g_{m \cdot m}}{g_{m \cdot a}} .
$$

Clearly, there exists a unique coupler ratio $k_{\max }$ for which the required generalized Doherty operation specified by (25) is exactly achieved by the auxiliary transistor operating in class $\mathrm{C}$ at both $\theta=0$ (back-off) and $\theta_{\max }$ (peak power).

\section{ACKNOWLEDGMENT}

The authors would like to thank the anonymous reviewers for in depth feedback and invaluable suggestions for improving the manuscript.

\section{REFERENCES}

[1] W. H. Doherty, "A new high efficiency power amplifier for modulated waves," Proc. IRE, vol. 24, pp. 1163-1182, Sep. 1936

[2] J. Xia, X. Zhu, L. Zhang, J. Zhai, and Y. Sun, "High-efficiency GaN Doherty power amplifier for $100-\mathrm{MHz}$ LTE-advanced application based on modified load modulation network," IEEE Trans. Microw. Theory Techn., vol. 61, no. 8, pp. 2911-2921, Aug. 2013.

[3] M. Iwamoto, A. Williams, P. Chen, A. G. Metzger, L. E. Larson, and P M. Asbeck, "An extended Doherty amplifier with high efficiency over a wide power range," IEEE Trans. Microw. Theory Techn., vol. 49, no. 12, pp. 2472-2479, Dec. 2001.

[4] J. Son, I. Kim, J. Moon, J. Lee, and B. Kim, "A highly efficient asymmetric Doherty power amplifier with a new output combining circuit," in Proc. IEEE Int. Microw., Commun., Antennas Electron. Syst. Conf., Tel Aviv, Israel, Nov. 2011, pp. 1-4.

[5] H. Jang, P. Roblin, and C. Quindroit, "Adjustable load-modulation asymmetric Doherty amplifier design using nonlinear embedding," in IEEE MTT-S Int. Microw. Symp. Dig., Tampa, FL, USA, Jun. 2014, pp. $1-4$.

[6] D. Y. Wu, J. Annes, M. Bokatius, P. Hart, E. Krvavac, and G. Tucker, "A $350 \mathrm{~W}, 790$ to $960 \mathrm{MHz}$ wideband LDMOS Doherty amplifier using a modified combining scheme," in IEEE MTT-S Int. Microw. Symp. Dig., Tampa, FL, USA, Jun. 2014, pp. 1-4.

[7] H. Jang, P. Roblin, and Z. Xie, "Model-based nonlinear embedding for power amplifier design," IEEE Trans. Microw. Theory Techn., vol. 62, no. 9, pp. 1986-2002, Sep. 2014.

[8] A. Raffo, F. Scappaviva, and G. Vannini, "A new approach to microwave power amplifier design based on the experimental characterization of the intrinsic electron-device load line," IEEE Trans. Microw. Theory Techn., vol. 57, no. 7, pp. 1743-1752, Jul. 2009.

[9] A. Musio, V. Vadalà, F. Scappaviva, A. Raffo, S. Di Falco, and G. Vannini, "A new approach to class-E power amplifier design," in Proc. Workshop Integr. Nonlinear Microw. and Millimeter-Wave Circuits, 2011, pp. 1-4.
[10] S. Liu and D. M. M.-P. Schreurs, "Intrinsic class-F RF GaN power amplifier with commercial transistor based on a modified "hybrid" approach," in Proc. Workshop Integr. Nonlinear Microw. Millimeter-Wave Circuits, 2012, pp. 1-3.

[11] D. M. M.-P. Schreurs, J. Verspecht, S. Vandenberghe, and E. Vandamme, "Straightforward and accurate nonlinear device model parameter-estimation method based on vectorial large-signal measurements," IEEE Trans. Microw. Theory Techn., vol. 50, no. 4, pp. 2315-2319, Apr. 2002.

[12] G. Avolio, D. M. M.-P. Schreurs, A. Raffo, G. Crupi, G. Vannini, and B. Nauwelaers, "Waveforms-only based nonlinear de-embedding in active devices," IEEE Microw. Wireless Compon. Lett., vol. 22, no. 4, pp. 215-217, Dec. 2012.

[13] V. Vadalà, A. Raffo, S. Di Falco, G. Bosi, A. Nalli, and G. Vannini, "A loadpull characterization technique accounting for harmonic tuning," IEEE Trans. Microw. Theory Techn., vol. 61, no. 7, pp. 2695-2704, Jul. 2013.

[14] V. Vadalà, G. Avolio, A. Raffo, D. M. M.-P Schreurs, and G. Vannini, "Nonlinear embedding and de-embedding techniques for large-signal FET measurements," Microw. Opt. Technol. Lett., vol. 54, no. 12, pp. 2835-2838, Dec. 2012.

[15] B. Kim, I. Kim, and J. Moon, "Advanced Doherty architecture," IEEE Microw. Mag., vol. 11, no. 5, pp. 72-86, Aug. 2010.

[16] R. Darraji, F. M. Ghannouchi, and O. Hammi, "A dual-input digitally driven Doherty amplifier architecture for performance enhancement of Doherty transmitters," IEEE Trans. Microw. Theory Techn., vol. 59, no. 5, pp. 1284-1293, May 2011.

[17] T. M. Hone, S. Bensmida, K. A. Morris, M. A. Beach, J. P. McGeehan, J. Benedikt, and P. J. Tasker, "Controlling active load-pull in a dualinput inverse load modulated Doherty architecture," IEEE Trans. Microw. Theory Techn., vol. 60, no. 6, pp. 1797-1804, Jun. 2012.

[18] M. Naseri-Ali-Abadi, H. Golestaneh, H. Sarbishaei, and S. Boumaiza, "An extended bandwidth Doherty power amplifier using a novel output combiner," in IEEE MTT-S Int. Microw. Symp. Dig., Tampa, FL, USA, Jun. 2014, pp. 1-4.

[19] R. Giofre, L. Piazzon, P. Colantonio, and F. Giannini, "A distributed matching/combining network suitable to design Doherty power amplifiers covering more than an octave bandwidth," in IEEE MTT-S Int. Microw. Symp. Dig., Tampa, FL, Jun. 2014, pp. 1-3.

[20] X. Nghiem, J. Guan, and R. Negra, "Design of a broadband three-way sequential Doherty power amplifier for modern wireless communications," in IEEE MTT-S Int. Microw. Symp. Dig., Tampa, FL, USA, Jun. 2014, pp. 1-4.

[21] F. H. Raab, "Efficiency of Doherty RF power-amplifier systems," IEEE Trans. Broadcasting, vol. BC-33, no. 3, pp. 77-83, Sep. 1987.

[22] R. Raich, H. Qian, and G. T. Zhou, "Orthogonal polynomials for power amplifier modeling and predistorter design," IEEE Trans. Veh. Technol., vol. 53, no. 5, Sep. 2004.

[23] C. Quindroit, N. Naraharisetti, P. Roblin, S. Gheitanchi, V. Mauer, and M. Fitton, "FPGA implementation of orthogonal 2D digital predistortion system for concurrent dual-band power amplifiers based on time-division multiplexing," IEEE Trans. Microw. Theory Techn., vol. 61, no. 12, pp. 4591-4599, Dec. 2013.

[24] S. C. Cripps, RF Power Amplifiers for Wireless Communications, 2nd ed. Norwood, MA, USA: Artech House, 2006.

[25] S. Di Falco, A. Raffo, V. Vadalà, and G. Vannini, "Power amplifier design accounting for input large-signal matching," in Proc. Eur. Microw. Integr. Circuits Conf., Amsterdam, The Netherlands, Oct. 2012, pp. 465-468.

[26] A. Ramadan, T. Reveyrand, A. Martin, J. Nebus, P. Bouysse, L. Lapierre, J. Villemazet, and S. Forestier, "Two-stage GaN HEMT amplifier with gate-source voltage shaping for efficiency versus bandwidth enhancements," IEEE Trans. Microw. Theory Techn., vol. 59, no. 3, pp. 699-706, Mar. 2011.

[27] M. Haynes, S. C. Cripps, J. Benedikt, and P. J. Tasker, "PAE improvement using 2nd harmonic source injection at x-band," in Proc. Workshop Integr. Nonlinear Microw. Millimetre-Wave Circuits, 2012, pp. $1-3$.

[28] A. Dani, M. Roberg, and Z. Popovic, "PA efficiency and linearity enhancement using external harmonic injection," IEEE Trans. Microw. Theory Techn., vol. 60, no. 12, pp. 4097-4106, Dec. 2012.

[29] D. M. Pozar, Microwave Engineering, 3rd ed. Hoboken, NJ, USA: Wiley, 2005, ch. 4.5 . 


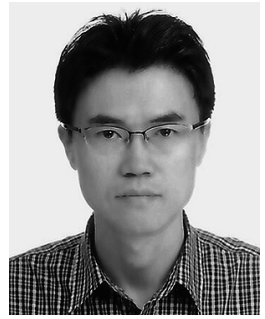

Haedong Jang (S'09-M'14) was born in Wonju, Korea, in 1971. He received the B.S. degree in electrical engineering from Kangwon National University, Chuncheon, Korea, in 1994, the M.S. and $\mathrm{Ph} . \mathrm{D}$. degrees in electrical and computer engineering from Inha University, Incheon, Korea, in 2005 and 2008 , respectively, and the Ph.D. degree in electrical and computer engineering from The Ohio State University, Columbus, OH, USA, in 2014.

He joined a nonprofit governmental organization, Small Business Corporation, Shiheung, Korea, in 1996, where he served as a Product Development Assistant Consultant until 2007 and was involved in more than 100 commercialized consumer product developments. He joined Infineon Technologies North America Corporation, Morgan Hill, CA, USA, as an Advanced Development RF Engineer in 2014. His main interests include advanced RF power amplifier architecture, nonlinear devices characterization, modeling, and power amplifier linearization.

Dr. Jang was a corecipient of the First Place Prize at the 2012 IEEE Microwave Theory and Techniques Society (IEEE MTT-S) International Microwave Symposium (IMS) student development of a Large-Signal-Network-Analyzer-Round-Robin Design Competition.

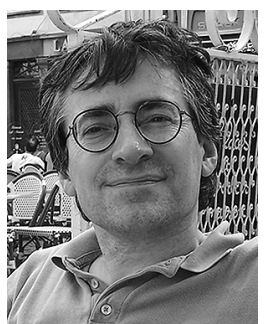

Patrick Roblin (M'85) was born in Paris, France, in September 1958. He received the Maitrise de Physics degree from the Louis Pasteur University, Strasbourg, France, in 1980, and the M.S. and D.Sc. degrees in electrical engineering from Washington University, St. Louis, MO, USA, in 1982 and 1984, respectively.

In 1984, he joined the Department of Electrical Engineering, The Ohio State University (OSU), Columbus, OH, USA, as an Assistant Professor and is currently a Professor. He is the first author of a textbook titled High-Speed Heterostructure Devices (Cambridge University Press, 2002). He is the founder of the Non-Linear RF Research Laboratory at OSU. At OSU, he has developed two educational RF/microwave laboratories and associated curriculum for training both undergraduate and graduate students. His present research interests include the measurement, modeling, design and linearization of nonlinear RF devices and circuits such as oscillators, mixers, and power-amplifiers.

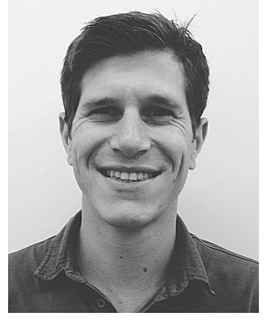

Christophe Quindroit was born in Corbeil-Essonnes, France, in October 1982. He received the M.Tech. and M.S. degrees in electronics from the Ecole Polytechnique de l'Université de Nantes, Nantes, France, in 2005, and the Ph.D. degree in electronics from the University of Limoges, Limoges, France, in 2010.

He was a Project Engineer with Alcatel-Lucent, France. He was a Research Engineer with The Ohio State University, Columbus, OH, USA. He is currently a Radio Engineer with LPA Concepts, Martillac, France. His research interests include analog system-level modeling, PA linearization techniques, and FPGA implementation.

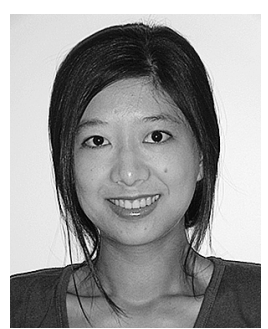

Yiqiao Lin is currently working toward the Ph.D. degree at The Ohio State University, Columbus, $\mathrm{OH}$, USA.

Her current research interests are in microwave circuit design for wireless communication systems. Her current work focuses on the development of concurrent dual-band envelope tracking power amplifiers for base stations.

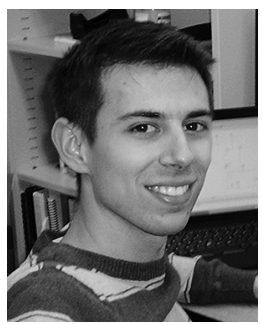

Robert D. Pond received the B.S. degree in electrical engineering from The Ohio State University, Columbus, OH, USA, in 2013, where he is currently working toward the M.S. degree in electrical engineering under the advisement of Prof. Patrick Roblin.

His research interests include design of ultra-wideband, high-efficiency, monolithic microwave integrated circuit power amplifiers. 